\title{
LOS VISITANTES DEL PARC NATURAL DE L'ALT PIRINEU Y LA PRÁCTICA DE ACTIVIDADES RECREATIVO-DEPORTIVAS. UNA PROPUESTA DE SEGMENTACIÓN
}

\section{Visitors to Alt Pirineu Natural Park and the practice of recreational and sports activities. A segmentation proposal}

\author{
E. I. Farías $^{1 *} \&$ S. Monserrat ${ }^{2}$ \\ ${ }^{1}$ Consultoría Socioambiental Ecogestión (Lleida) y GISEAFE Afores s/n. Castelldans - 25154. Lleida \\ ${ }^{2} \mathrm{HE}$ Sport Management- Loughborough College (UK) \\ *ecogestion@cag.es
}

Recibido: 07-10-2013. Aceptado: 15-11-2013. Fecha de publicación on-line: 21-10-2014

Citation / Cómo citar este artículo: Farías, E. I. \& Monserrat, S. (2014). Los visitantes del Parc Natural de l'Als Pirineu y la práctica de actividades recreativo-deportivas. Una propuesta de segmentación. Pirineos, 169, e005. doi. http://dx.doi. org/10.3989/Pirineos.2014.169005

RESUMEN: En los últimos años se ha registrado un gran incremento en la práctica de las actividades recreativodeportivas en los espacios naturales protegidos. En el marco de la planificación y la gestión del uso público de este tipo de espacios, resulta fundamental profundizar en el conocimiento de las características de los visitantes. En este estudio se analiza el perfil de los diferentes segmentos de visitantes del Parc Natural de l'Alt Pirineu en base a las actividades recreativo-deportivas practicadas. Para este estudio se realizaron 1.600 encuestas en 16 puntos de muestreo, a lo largo de un año natural comprendido entre los meses de Julio de 2010 y Junio de 2011. En total se identificaron ocho segmentos de visitantes en función de la actividad física: paseantes, excursionistas, senderistas, montañistas, ciclistas, recolectores de setas, motoristas y esquiadores. Los resultados aquí obtenidos son discutidos en términos de su posible aplicación en la gestión del uso público de este parque (extrapolable también a otros espacios naturales protegidos españoles), proponiendo estrategias de gestión y campañas de sensibilización dirigidas a los diferentes segmentos de visitantes.

PALABRAS CLAVE: Actividades recreativo-deportivas; segmentación de visitantes; espacios naturales protegidos

\begin{abstract}
In recent years there has been a large increase in the practice of recreational and sports activities in protected natural areas. As part of the planning and management of public use of these areas, is essential to a deeper understanding of the characteristics of the visitors. This study analyzes the characteristics of the different segments of visitors to Alt Pirineu Natural Park based on recreational and sports activities practiced. For this study, 1.600 surveys were conducted in 16 sampling points over a calendar year between the months of July 2010 and June 2011. In total we identified eight segments of visitors based on physical activity: walkers, recreational hikers, hikers, mountaineer, cyclists, mushroom picker, off road driver and skier. The results obtained here are discussed in terms of their possible applicability in the management of public use of this park and other protected natural areas and also for the development of management strategies and awareness campaigns aimed at different segments of visitors.
\end{abstract}

KEYWORDS: Recreational and sports activities; visitor segmentation; protected natural areas 


\section{Introducción}

Los espacios naturales protegidos, como el que nos ocupa, cumplen con importantes funciones ambientales, como pueden ser: mantener la diversidad biológica, conservar zonas productoras de agua, alojar una gran número de especies animales y vegetales; pero también, desde un punto de vista más social, son enclaves fundamentales para el desarrollo de la cada vez más creciente demanda de práctica de actividades recreativo-deportivas en el medio natural.

De acuerdo con los datos publicados por EuroparcEspaña (Europarc-España, 2008), en el año 2006 el conjunto de espacios naturales protegidos de España recibió más de 36 millones de visitantes, alrededor de un 4,5\% más que en años previos. La Comunidad autónoma de Catalunya fue una de las más visitadas: 8 millones de personas visitaron uno de los 17 parques naturales catalanes pertenecientes a red Natura 2000, entre los que se encuentra el Parque Natural de l'Alt Pirineu (PNAP). Así mismo, estudios como los llevados a cabo por Múgica (1993), Farías (2000), Atauri et al. (2000), Muñoz (2008), Farías et al. (2010), OAPN (2011), entre otros, también alertan sobre la presencia de una gran diversidad de usos, entre los que cabe destacar aquellos que implican la práctica de actividades físico-deportivas. Según estos autores, entre el $50 \%-60 \%$ de los visitantes que concurren a estos espacios realizan algún tipo de actividad físico-deportiva durante su visita, siendo en este caso la actividad del senderismo, unas de las más practicadas.

Con respecto a ello, son numerosos los documentos y autores que sostienen que el incremento de la presión y diversidad de la demanda recreativa, deportiva y naturalística de estos espacios es uno de los aspectos más dinámicos en cuanto a los cambios de uso producidos en los mismos y en sus áreas de influencia. De aquí, que la planificación y gestión de dichas actividades, conocidas en el ámbito de la gestión de estos espacios como uso público (léase: conjunto de prácticas y actividades que se derivan del uso y el disfrute por parte de las personas que visitan los espacios naturales protegidos, de manera individual o colectiva, de forma espontánea u organizada, con la finalidad principal de disfrutar de sus valores naturales, ambientales, estéticos, paisajísticos o culturales; OAPN, 2011), se haya convertido en los últimos años en uno de los principales retos para los responsables de la gestión de los espacios naturales protegidos, y a la vez, en una gran oportunidad para intentar introducir mejoras en la promoción de un modelo de turismo más sostenible y más compatible con los objetivos de gestión de los territorios protegidos (Wall Reinius \& Fredman, 2007).

En este sentido, y a pesar de que numerosos investigadores y/o profesionales del tema defienden que la planificación ha de constituirse como el primer paso en la programación de cualquier tipo de actuación de uso público (Benayas, 2000; Newsome et al., 2002; Gómez-Limón, 1996; Gómez-Limón et al., 2002; Gruber \& Benayas, 2002; Hockings et al., 2002, etc.), en demasiadas ocasiones ésto no sucede así. La presión o cambios de la demanda, la falta de recursos necesarios o las prisas en la solución de determinadas problemáticas, hacen que se ignore este trabajo de planificación y se desarrolle una gestión más de tipo adaptativa, en donde las decisiones se toman a partir de la experiencia, del ensayo-error o del seguimiento de los resultados conseguidos. No es una tarea sencilla tratar de prevenir o disminuir los impactos ambientales negativos sin perjudicar la calidad de las experiencias recreativas de los visitantes (tan importante a la hora de valorar la función educativa y divulgativa de estos espacios). Se trata de optimizar la gestión de este tipo de usos, a la vez que se cumplen los objetivos de conservación, desarrollo y aprovechamiento recreativo de estos espacios naturales. De aquí la importancia de facilitar a los gestores una información lo más sistematizada posible sobre el uso actual en los espacios naturales protegidos.

Son diversos los autores que abogan por la incorporación de la segmentación del mercado en los sistemas de gestión de este tipo de espacios (Frochot, 2005; Chi-Ok \& Ditton, 2006; Schmitz et al., 2007; Schuett \& Pierskalla, 2007; Arnberger et al., 2012), siendo abundantes los trabajos desarrollados en este sentido (Brown \& Haas, 1980; Collins \& Hodge, 1984; Schreyer, 1982; Rollins \& Rouse, 1992; Kuentzel \& Heberlein, 1992; McColl \& Reilly 1993; De Lucio \& Múgica, 1994; Merrill \& Graefe, 1998; Virden \& Schreyer, 1998; Bricker \& Kerstetter, 2000; Farías et al., 2005; Schuett \& Pierskalla, 2007; Woratschek et al., 2007; Farías 2011a).

Sin embargo, la aplicación práctica de este tipo de estudios puede ser problemática, sobre todo a la hora de intentar identificar o reproducir los segmentos a posteriori con el fin de realizar un seguimiento o diseñar estrategias de gestión. Según Kotler (1994), un segmento de mercado debe ser claramente diferenciable de otros segmentos sobre la base de algunas características que sostengan su homogeneidad interna; al tiempo que debe ser accesible, medible y apropiado para los objetivos que se pretender abordar.

Cabe destacar que estudios previos han demostrado que las necesidades, preferencias y comportamientos de los visitantes de este tipo de parques suelen diferenciarse en base a la actividad realizada (Priskin, 2003; Cessford, 2003; Pouta et al., 2004); concretamente cuanto más activa es la práctica realizada, más uniforme es el perfil de los visitantes, más claras sus preferencias y comportamientos $\mathrm{y}$, por lo tanto, más fácil su identificación (Mowen et al., 2012).

Así pues, en este trabajo se analizan parte de los resultados obtenidos en el Estudio de Afluència i Caracterització dels Visitants del Parc Natural de l'Alt Pirineu (2011), con la finalidad de proponer una segmentación de los visitantes del Parc Natural de l'Alt Pirineu en base a las actividades recreativo-deportivo practicadas. Creemos que esta propuesta de segmentación posee una gran aplicabilidad y potencial estratégico a la hora de gestionar la creciente y, cada vez más diversificada, demanda de este grupo de actividades en este tipo de espacios. 


\section{Metodología}

\subsection{Contexto de la investigación}

El presente estudio se llevó a cabo en el Parque Natural de l'Alt Pirineu, siendo éste el más grande de Cataluña. El parque fue establecido en 2003 por la Generalitat de Catalunya y ocupa una extensión de 69.850 hectáreas a lo largo de las comarcas del Pallars Sobirà y Alt Urgell, e incluye algunos de los picos más altos del Pirineo Catalán (Figura 1). Dentro de la clasificación de la UICN (Dudley, 2008) está considerado como categoría V- paisajes terrestres/marinos protegidos (Protected Landscape/ Seascape).

Desde un punto de vista geográfico, el PNAP se encuentra dividido en cinco grandes sectores: Valls d'Àneu, Vall de Cardós, Vall Ferrera y Vall de Santa Magdalena y Massís de l'Orri. Todos ellos con un importante valor ecológico y paisajístico que combinan de una forma ejemplar la diversidad de paisajes propios de la alta y media montaña pirenaica. Existe además un extenso patrimonio cultural, fruto de la histórica acción humana sobre el territorio. En este sentido, una de las características más importantes de este Parque Natural es que cuenta con una amplia provisión de itinerarios y de áreas gestionadas aptas para la práctica de actividades como el senderismo, el montañismo, la bicicleta de montaña o la conducción de vehículos $4 \times 4$. La oferta recreativa del parque incluye más de 173 itinerarios (para más información consultar Farías, 2011b).

\subsection{Metodología de investigación}

Los datos considerados en este estudio se basaron en la realización de un total de 1.600 encuestas distribuidas proporcionalmente en 16 puntos de acceso o muestreo, de acuerdo a la afluencia de visitantes registrada durante la realización del trabajo: a mayor afluencia de visitantes, mayor número de encuestas.

Las encuestas se llevaron a cabo a lo largo del año natural comprendido entre Julio del 2010 y Junio del 2011. Se realizaron entre 6 y 14 días de trabajo de campo por cada punto de muestreo (laborales y festivos), dependiendo de la categoría de uso asignada y las características principales de los mismos, tal como sigue: a) punto de acceso prioritario o de uso máximo a lo largo del año; b) punto de acceso secundario o de uso ocasional durante el verano; inaccesible durante los meses de invierno, etc. Los datos fueron recogidos en un total de 133 días de trabajo de campo (Tabla 1).

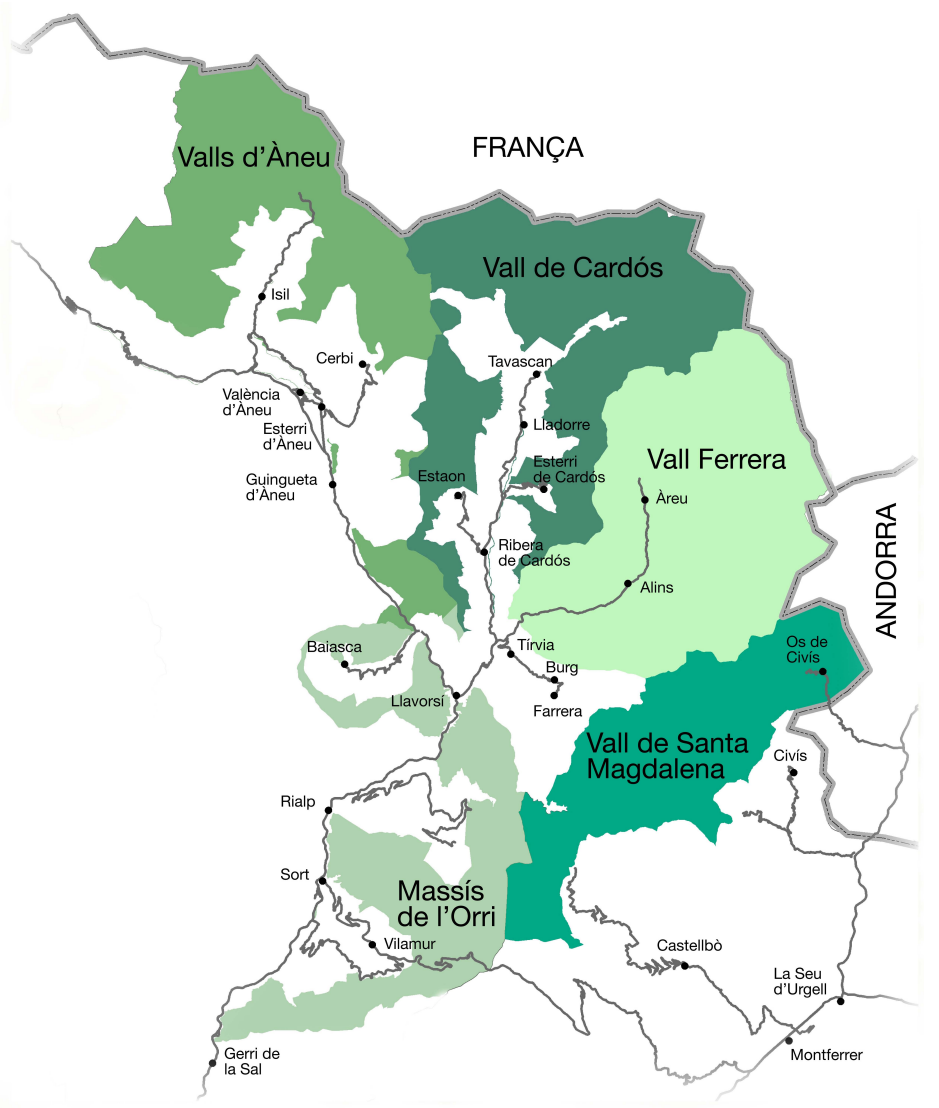

Figura 1. Parque Natural de l'Alt Pirineu. Fuente: PNAP Figure 1. Alt Pirineu Natural Park. Source: FNAP 


\section{• E. I. FARÍAS \& S. MONSERRAT}

Tabla 1. Puntos de acceso controlados y distribución de días y número de encuestas realizadas Table 1. Sampling distribution and surveying days for each access point

\begin{tabular}{|c|c|c|c|c|c|c|}
\hline Punto de muestreo & Ubicación & $\begin{array}{l}\text { Tipo de } \\
\text { acceso }\end{array}$ & $\begin{array}{c}\text { Días } \\
\text { laborales }\end{array}$ & $\begin{array}{c}\text { Días } \\
\text { festivos }\end{array}$ & Total días & $\begin{array}{l}\text { Número de } \\
\text { encuestas }\end{array}$ \\
\hline 1. Fornet & Valls d'Àneu & prioritario & 5 & 4 & 9 & 123 \\
\hline 2. Cerbi & Valls d'Àneu & prioritario & 4 & 4 & 8 & 58 \\
\hline 3. Tavascan & Vall de Cardós & prioritario & 10 & 5 & 15 & 405 \\
\hline 4. Esterri de Cardós & Vall de Cardós & prioritario & 6 & 4 & 10 & 63 \\
\hline 5. La Farga & Vall Ferrera & prioritario & 6 & 4 & 10 & 185 \\
\hline 6. Tor & Vall Ferrera & prioritario & 6 & 4 & 10 & 101 \\
\hline 7. Bordes de Virós & Vall Ferrera & prioritario & 9 & 5 & 14 & 105 \\
\hline 8. Coll de So & Vall de Sta. Magdalena & prioritario & 6 & 4 & 10 & 44 \\
\hline 9. Sant Joan de l'Erm & Vall de Sta. Magdalena & prioritario & 11 & 6 & 17 & 268 \\
\hline 10. Baiasca & Massís de l'Orri & prioritario & 6 & 4 & 10 & 75 \\
\hline 11. Os de Civís & Vall de Sta. Magdalena & prioritario & 2 & 4 & 6 & 71 \\
\hline 12. Comes de Rubió & Massís de l'Orri & prioritario & 3 & 3 & 6 & 29 \\
\hline 13. Mollera & Valls d'Àneu & secundario & 1 & 1 & 2 & 33 \\
\hline 14. Escaló & Valls d'Àneu & secundario & 1 & 1 & 2 & 13 \\
\hline 15. Estaon & Vall de Cardós & secundario & 1 & 1 & 2 & 10 \\
\hline \multirow[t]{2}{*}{ 16. Arboló } & Massís de l'Orri & secundario & 1 & 1 & 2 & 17 \\
\hline & & & 80 & 51 & 133 & 1.600 \\
\hline
\end{tabular}

Teniendo en cuenta que la mayor parte de las preguntas incluidas en el cuestionario hacían referencia a hechos ya consumados (ej.: itinerario visitado, valoración de ciertos servicios, duración del tiempo de visita, opinión sobre los servicios consumidos, etc.), la totalidad de las encuestas fueron realizadas en el momento en que los visitantes abandonaban el parque por alguno de los accesos controlados, dentro de la franja horaria de 12:00 h a 19:00 h (durante los meses de primavera-verano) o de 12:00 a 17:00 h. (durante los meses de otoño-invierno).

Para la realización de las encuestas se contó con la colaboración de personal capacitado y formado para tal fin. Este personal está compuesto por: seis informadores del parque, cuatro estudiantes de la Universidad de Lleida (ETSEA i INEFC) y un grupo de nueve personas contratadas para tal fin.

El sistema de selección de los encuestados fue aleatorio, de acuerdo a las características básicas de cada punto de acceso. Por ejemplo, se realizó una encuesta por vehículo en los accesos con uso mayoritario de coches, una encuesta cada tres grupos de caminantes o ciclistas, en todos los accesos, o sólo dos encuestas por grupo, en el caso de grupos de más de 10 personas. El ratio de respuesta final ascendió al $90 \%$ y el tiempo medio empleado en la realización de las diferentes encuestas rondó los 5 minutos.

El modelo de encuesta utilizado incluyó 26 preguntas cerradas, organizadas en 3 secciones claramente diferenciadas: sección $1^{\mathrm{a}}$ - preguntas de tipo demográficas (6 pre- guntas), incluido aspectos tales como: lugar de residencia habitual, ocupación, nivel de formación, género, edad y conocimientos sobre el status de la protección del espacio; sección $2^{\mathrm{a}}$ - preguntas relacionadas con las características de visita (9 preguntas): medios por los cuales conoció la existencia de este Parque Natural, grupo de visita, número de integrantes por grupo, duración de la estancia en el parque, alojamiento, frecuencia de visita, medio de transporte utilizado para acceder al parque, motivaciones de visita, actividad practicada y lugar visitado (incluido itinerario/zona); y sección $3^{\text {a }}$ - preguntas relativas a las opiniones y sugerencias de mejora (11 preguntas): experiencia de conflicto, opiniones sobre la masificación de la zona, señalización, limpieza, infraestructuras, mantenimiento o conservación del PNAP, necesidades de nuevas infraestructuras o servicios, regulación de la recolección de setas y circulación de vehículos motorizados y aspecto a mejorar.

En cuanto a las motivaciones de visita, se incluyeron aquellos indicadores más destacables en base a la consideración de trabajos previos como los elaborados por McColl \& Reilly (1993), Wallace \& Smith (1997) y Chhetri et al., (2004), Farías et al., (2005). En esta pregunta, al igual que en la relativa a la necesidad de nuevos servicios, los encuestados podían responder hasta un máximo de tres opciones, previa asignación de un orden de prioridad.

Con el fin de facilitar la comprensión y tratamiento estadístico de la frecuentación de los diferentes itinerarios- 
zonas, estos fueron categorizados en base al nivel de popularidad y accesibilidad: 1) Rutas clásicas, itinerarios característicos de cada zona, reseñados en los mapas del parque y, por lo tanto, mejor señalizados y 2) Rutas no clásicas, resto de senderos no incluidos en la primera categoría. Además se añadió la opción no visita Parque, por el hecho de no haber considerado a priori esta posibilidad.

Finalmente hay que destacar, en cuanto a terminología utilizada en la sistematización de las actividades recreativo-deportivas incluida en la encuesta (Farías, 2000, Farías et al., 2010; Farías, 2011a, etc.), la consideración de las siguientes categorías: 1) excursionismo, actividad físico-deportiva que consiste en caminar por el campo, el bosque o las montañas a lo largo de senderos o caminos durante menos de 1 hora, 2) senderismo, ídem pero por un tiempo superior a una hora y 3) montañismo, que se diferencia de las dos anteriores por el hecho de requerir la ascensión obligatoria de un pico. Así mismo, para una mayor facilidad en la interpretación de los datos, las actividades de raquetas de nieve, esquí nórdico, esquí de montaña y esquí alpino, fueron agrupadas en un solo grupo de actividades como actividades de nieve.

\subsection{Análisis de los datos}

El tratamiento estadístico de los datos se llevó a cabo mediante el procesador estadístico PASW (SPSS Statistics 18). En primer lugar, se realizó un análisis estadístico descriptivo de las 1.600 encuestas, con el fin de tener una visión general del nivel de práctica de las diferentes actividades recreativo-deportivas registradas durante el tra- bajo de campo. En segundo lugar, una vez identificados los ocho grandes grupos de actividades y un grupo residual de 46 casos variopintos (incluidas actividades minoritarias como la pesca de trucha, la escalada en roca, etc.), se procedió a aplicar los análisis estadísticos no paramétricos como el test de Chi-Cuadrado y Kruskal-Wallis, de acuerdo al tipo de variables analizadas (nominales o categóricas). Los datos obtenidos fueron diferenciados en base a dos niveles de significación: $<0.01 \mathrm{y}<0.05$ (Siegel \& Castellan 1988).

\section{Resultados}

\subsection{Actividades recreativo-deportivas y perfil medio de los visitantes del Parque Natural}

Los resultados obtenidos respecto a las actividades practicadas muestran la presencia de una práctica mayoritaria de las actividades del excursionismo $(18,1 \%)$, el senderismo $(27,3 \%)$ y la recolección de setas $(12,4 \%)$. Así mismo, destaca el alto nivel de práctica deportiva entre los visitantes, alrededor de un 70\% (excluyendo la permanencia en el lugar de llegada o la conducción de vehículos a motor - Tabla 2), un 10\% más que en estudios previos (Múgica, 1993; Muñoz, 2008; OAPN, 2011; Farías, 2011a).

Así mismo, el perfil medio del visitante del PNAP es: hombre $(66,9 \%)$, con una media de edad de 44,8 años ( $29 \%$ entre 32 y 41 años), trabajador por cuenta ajena $(44,7 \%)$, residente en la provincia de Barcelona $(56,7 \%)$, poseedor de estudios de nivel universitario $(48,9 \%)$ y co-

Tabla 2. Actividades recreativo-deportivo-recreativas al Parc Natural de l'Alt Pirineu Table 2. Recreational and physical activities in the Alt Pirineu Natural Park

\begin{tabular}{|l|r|r|}
\hline \multicolumn{1}{|c|}{ Actividades deportivo-recreativas } & \multicolumn{1}{c|}{ Número de visitantes } & \multicolumn{1}{c|}{ Porcentajes } \\
\hline Permanecer en lugar de llegada & 135 & 8,4 \\
\hline Excursionismo (menos de 1 h) & 289 & 18,1 \\
\hline Senderismo (más de 1 h) & 436 & 27,3 \\
\hline Montañismo (ascensión pico) & 107 & 6,7 \\
\hline Bicicleta de montaña & 68 & 4,3 \\
\hline Recolección de setas & 199 & 12,4 \\
\hline Rutas 4x4 en coche & 109 & 6,8 \\
\hline Rutas con quads, motos & 22 & 1,4 \\
\hline Raquetas de nieve & 36 & 2,3 \\
\hline Esquí nórdico & 81 & 5,1 \\
\hline Esquí alpino & 54 & 3,4 \\
\hline Esquí de montaña & 18 & 1,1 \\
\hline Pesca truchas en lagos & 20 & 1,3 \\
\hline Otras & 26 & 1,6 \\
\hline Total muestra & 1600 & 100.0 \\
\hline
\end{tabular}


nocedor del estatus de protección del parque $(83,6 \%$ sabe que el PNAP es un espacio natural protegido). Visita el parque durante medio día $(71,8 \%$, con un valor medio de 6,95 horas), con una media de casi 4 personas por grupo y principalmente lo hace durante los meses de Julio $(12,1 \%)$, Agosto $(30,6)$ y Septiembre $(14,0 \%)$. Se aloja mayoritariamente en segundas residencias $(17,2 \%)$, hoteles $(17,9 \%)$ y campings $(13,6 \%)$, accede al parque en vehículo particular $(84,5 \%)$ y por primera vez $(34,9 \%)$. El motivo principal de la visita es Estar cerca de la naturaleza $(28,1 \%)$, Practicar alguna actividad fisica (17,9\%) o Relajarse y desconectar (14,4\%). Además, suele escoger un itinerario clásico para adentrarse en el parque $(78,3 \%)$ y no acostumbra a visitar otras zonas del parque (52,9\%; Tablas 3 y 4).

Por término medio, el visitante tipo está satisfecho con su visita al parque ya que evalúa positivamente la mayor parte de los aspectos valorados, como el grado de masificación de personas (93,3\% opina que no hay), la vivencia de algún tipo de conflicto (el 64,3\% manifestó no haber experimentado ningún tipo de conflicto con otras personas, usos, etc.), el estado de limpieza (87,9\% opina que está muy correcto o correcto), de las infraestructuras $(72,7 \%$ ídem) y la señalización (72,5\% ídem). Así mismo, algunos de los servi-

Tabla 3. Variables que mejor definen las características sociológicas de los grupos de visitantes (ver texto para más detalles).

Los valores son porcentajes del número de sujetos por cada grupo.

Table 3. Variables that best define the sociological characteristics of visitor groups (see text for more details).

Values are percentages of the number of subjects for each group

\begin{tabular}{|c|c|c|c|c|c|c|c|c|c|}
\hline Variables & $\begin{array}{c}\text { Paseantes } \\
\mathrm{n}=135\end{array}$ & $\begin{array}{c}\text { Excursionistas } \\
\mathbf{n}=\mathbf{2 8 9}\end{array}$ & $\begin{array}{c}\text { Senderistas } \\
n=436\end{array}$ & $\begin{array}{c}\text { Montañistas } \\
\mathbf{n}=\mathbf{1 0 7}\end{array}$ & $\begin{array}{c}\text { Ciclistas } \\
n=68\end{array}$ & $\begin{array}{c}\text { Recolectores } \\
\text { de setas } \\
\mathbf{n}=199\end{array}$ & $\begin{array}{l}\text { Motoristas } \\
n=131\end{array}$ & $\begin{array}{c}\text { Esquiadores } y \\
\text { otras } \\
n=189\end{array}$ & $\begin{array}{c}\text { Total } \\
\text { Muestra }\end{array}$ \\
\hline $\begin{array}{l}\text { Grupos de edad* } \\
17 \text { a } 21 \text { años } \\
22 \text { a } 31 \text { años } \\
32 \text { a } 41 \text { años } \\
42 \text { a } 51 \text { años } \\
52 \text { a } 61 \text { años } \\
\text { Más } 62 \text { años }\end{array}$ & $\begin{array}{r}1,5 \% \\
13,3 \% \\
34,1 \% \\
27,4 \% \\
17,8 \% \\
5,9 \%\end{array}$ & $\begin{array}{r}1,0 \% \\
6,9 \% \\
28,0 \% \\
30,0 \% \\
20,1 \% \\
14,0 \%\end{array}$ & $\begin{array}{r}1,8 \% \\
14,4 \% \\
28,7 \% \\
28,0 \% \\
20,6 \% \\
7,0 \%\end{array}$ & $\begin{array}{r}2,8 \% \\
17,8 \% \\
38,2 \% \\
20,6 \% \\
18,7 \% \\
1,9 \%\end{array}$ & $\begin{array}{r}5,9 \% \\
23,5 \% \\
29,4 \% \\
32,0 \% \\
8,8 \% \\
0,0 \%\end{array}$ & $\begin{array}{r}0,5 \% \\
5,0 \% \\
17,1 \% \\
20,6 \% \\
35,2 \% \\
21,6 \%\end{array}$ & $\begin{array}{r}2,3 \% \\
7,6 \% \\
26,7 \% \\
28,3 \% \\
24,4 \% \\
10,7 \%\end{array}$ & $\begin{array}{r}1,6 \% \\
21,7 \% \\
38,1 \% \\
22,2 \% \\
11,1 \% \\
5,3 \%\end{array}$ & $\begin{array}{r}1,7 \% \\
12,6 \% \\
29,0 \% \\
26,4 \% \\
20,9 \% \\
9,4 \%\end{array}$ \\
\hline Media de edad (años) & 43,2 & 47,1 & 43,9 & 39,8 & 38,5 & 52,2 & 46,1 & 40,0 & 44,8 \\
\hline $\begin{array}{l}\text { Género* } \\
\text { Hombre } \\
\text { Mujer }\end{array}$ & $\begin{array}{l}58,5 \% \\
41,5 \%\end{array}$ & $\begin{array}{l}61,6 \% \\
38,4 \%\end{array}$ & $\begin{array}{l}64,0 \% \\
36,0 \%\end{array}$ & $\begin{array}{l}78,5 \% \\
21,5 \%\end{array}$ & $\begin{array}{l}82,4 \% \\
17,6 \%\end{array}$ & $\begin{array}{l}73,4 \% \\
26,6 \%\end{array}$ & $\begin{array}{l}82,4 \% \\
17,6 \%\end{array}$ & $\begin{array}{l}58,7 \% \\
41,3 \%\end{array}$ & $\begin{array}{l}66,9 \% \\
33,1 \%\end{array}$ \\
\hline $\begin{array}{l}\text { Lugar de residencia* } \\
\text { Provincia de Barcelona } \\
\text { Provincia de Lleida } \\
\text { Provincia de Tarragona } \\
\text { Provincia de Girona } \\
\text { Otras provincias } \\
\text { Francia } \\
\text { Otros países } \\
\text { Alrededores del parque } \\
\text { Andorra }\end{array}$ & $\begin{array}{r}54,9 \% \\
16,3 \% \\
9,6 \% \\
1,5 \% \\
5,9 \% \\
0,7 \% \\
0,7 \% \\
8,9 \% \\
1,5 \%\end{array}$ & $\begin{array}{r}61,9 \% \\
9,7 \% \\
5,2 \% \\
3,5 \% \\
6,6 \% \\
0,7 \% \\
1,7 \% \\
9,3 \% \\
1,4 \%\end{array}$ & $\begin{array}{r}58,7 \% \\
7,1 \% \\
3,2 \% \\
5,5 \% \\
12,8 \% \\
2,8 \% \\
4,8 \% \\
4,6 \% \\
0,5 \%\end{array}$ & $\begin{array}{r}57,9 \% \\
9,3 \% \\
8,4 \% \\
5,6 \% \\
9,3 \% \\
0,9 \% \\
1,9 \% \\
6,5 \% \\
1,9 \%\end{array}$ & $\begin{array}{r}57,4 \% \\
11,8 \% \\
2,9 \% \\
0,0 \% \\
8,8 \% \\
0,0 \% \\
2,9 \% \\
14,7 \% \\
1,5 \%\end{array}$ & $\begin{array}{r}51,8 \% \\
18,6 \% \\
7,0 \% \\
0,0 \% \\
0,5 \% \\
1,0 \% \\
0,0 \% \\
17,1 \% \\
4,0 \%\end{array}$ & $\begin{array}{r}50,9 \% \\
11,5 \% \\
6,1 \% \\
3,1 \% \\
4,6 \% \\
3,1 \% \\
3,1 \% \\
6,1 \% \\
11,5 \%\end{array}$ & $\begin{array}{r}55,1 \% \\
9,5 \% \\
7,4 \% \\
4,8 \% \\
5,3 \% \\
0,5 \% \\
0,5 \% \\
15,3 \% \\
1,6 \%\end{array}$ & $\begin{array}{r}56,7 \% \\
10,8 \% \\
5,6 \% \\
3,6 \% \\
7,3 \% \\
1,6 \% \\
2,1 \% \\
10,0 \% \\
2,3 \%\end{array}$ \\
\hline $\begin{array}{l}\text { Nivel educativo* } \\
\text { Educación primaria } \\
\text { Módulos profesionales } \\
\text { Educación secundaria } \\
\text { Universidad }\end{array}$ & $\begin{array}{r}6,7 \% \\
20,7 \% \\
24,4 \% \\
48,2 \%\end{array}$ & $\begin{array}{r}8,3 \% \\
17,6 \% \\
28,8 \% \\
45,3 \%\end{array}$ & $\begin{array}{r}6,0 \% \\
12,8 \% \\
23,2 \% \\
58,0 \%\end{array}$ & $\begin{array}{r}5,9 \% \\
7,4 \% \\
29,4 \% \\
57,3 \%\end{array}$ & $\begin{array}{r}5,6 \% \\
16,8 \% \\
21,5 \% \\
56,1 \%\end{array}$ & $\begin{array}{l}24,6 \% \\
16,6 \% \\
30,2 \% \\
28,6 \%\end{array}$ & $\begin{array}{r}8,4 \% \\
20,6 \% \\
22,9 \% \\
48,1 \%\end{array}$ & $\begin{array}{r}3,7 \% \\
15,9 \% \\
28,0 \% \\
52,4 \%\end{array}$ & $\begin{array}{r}8,8 \% \\
16,1 \% \\
26,2 \% \\
48,9 \%\end{array}$ \\
\hline $\begin{array}{l}\text { Ocupación* } \\
\text { Estudiante } \\
\text { Empleado cuenta ajena } \\
\text { Autónomo } \\
\text { Empresario } \\
\text { Funcionario } \\
\text { Desempleado } \\
\text { Jubilado } \\
\text { Ama de casa }\end{array}$ & $\begin{array}{r}0,7 \% \\
56,4 \% \\
14,8 \% \\
5,2 \% \\
13,3 \% \\
2,2 \% \\
6,7 \% \\
0,7 \%\end{array}$ & $\begin{array}{r}1,7 \% \\
41,2 \% \\
18,7 \% \\
5,9 \% \\
17,6 \% \\
3,5 \% \\
10,7 \% \\
0,7 \%\end{array}$ & $\begin{array}{r}5,9 \% \\
44,5 \% \\
14,9 \% \\
8,7 \% \\
17,7 \% \\
3,7 \% \\
4,1 \% \\
0,7 \%\end{array}$ & $\begin{array}{r}9,3 \% \\
51,5 \% \\
17,8 \% \\
5,6 \% \\
12,1 \% \\
2,8 \% \\
0,9 \% \\
0,0 \%\end{array}$ & $\begin{array}{r}11,8 \% \\
52,9 \% \\
11,8 \% \\
5,9 \% \\
10,3 \% \\
7,4 \% \\
0,0 \% \\
0,0 \%\end{array}$ & $\begin{array}{r}1,5 \% \\
41,3 \% \\
21,6 \% \\
8,0 \% \\
5,0 \% \\
5,5 \% \\
17,1 \% \\
0,0 \%\end{array}$ & $\begin{array}{r}2,3 \% \\
35,9 \% \\
26,7 \% \\
13,0 \% \\
8,4 \% \\
3,8 \% \\
9,9 \% \\
0,0 \%\end{array}$ & $\begin{array}{r}3,2 \% \\
47,5 \% \\
17,5 \% \\
6,9 \% \\
15,9 \% \\
5,3 \% \\
3,7 \% \\
0,0 \%\end{array}$ & $\begin{array}{r}3,9 \% \\
44,7 \% \\
18,0 \% \\
7,6 \% \\
13,8 \% \\
4,2 \% \\
7,4 \% \\
0,4 \%\end{array}$ \\
\hline $\begin{array}{l}\text { Conocimiento estatus } \\
\text { de protección } * * \\
\mathrm{Si}\end{array}$ & $81,4 \%$ & $86,2 \%$ & $85,8 \%$ & $92,5 \%$ & $75,0 \%$ & $76,3 \%$ & $78,5 \%$ & $81,5 \%$ & $83,6 \%$ \\
\hline
\end{tabular}

$\mathrm{n}=1554$

*Diferencias entre grupos con un nivel de significación de 0.05

**Diferencias entre grupos con un nivel de significación de 0.01 $n=1554$

* Differences between groups are significant at the 0.05 level

** Differences between groups are significant at the 0.01 level 
Tabla 4. Variables que mejor definen las características del viaje, el comportamiento y las motivaciones de visita (ver texto para más detalles). Los valores son porcentajes del número de sujetos por cada grupo.

Table 4. Variables that best define the groups'visiting behaviour and motivations of visitors (see text for more details).

Values are percentages of the number of subjects for each group

\begin{tabular}{|c|c|c|c|c|c|c|c|c|c|}
\hline Variables & $\begin{array}{c}\text { Paseantes } \\
\mathrm{n}=135\end{array}$ & $\begin{array}{c}\text { Excursionistas } \\
\mathbf{n}=\mathbf{2 8 9}\end{array}$ & $\begin{array}{c}\text { Senderistas } \\
n=436\end{array}$ & $\begin{array}{c}\text { Montañistas } \\
\mathbf{n}=\mathbf{1 0 7}\end{array}$ & $\begin{array}{c}\text { Ciclistas } \\
n=68\end{array}$ & $\begin{array}{c}\text { Recolectores } \\
\text { de setas } \\
\mathbf{n}=199\end{array}$ & $\begin{array}{c}\text { Motoristas } \\
\quad n=131\end{array}$ & $\begin{array}{c}\text { Esquiadores } \\
\text { y otras } \\
\mathbf{n}=189\end{array}$ & $\begin{array}{c}\text { Total } \\
\text { Muestra }\end{array}$ \\
\hline \multicolumn{10}{|l|}{$\begin{array}{l}\text { Medios de conocimiento } \\
\text { de la zona* }\end{array}$} \\
\hline Vivo en la zona & $9,6 \%$ & $8,0 \%$ & $6,4 \%$ & $4,7 \%$ & $8,8 \%$ & $15,6 \%$ & $6,9 \%$ & $15,3 \%$ & $9,6 \%$ \\
\hline No la conocía antes & $8,1 \%$ & $8,0 \%$ & $3,9 \%$ & $4,7 \%$ & $11,8 \%$ & $11,1 \%$ & $14,5 \%$ & $15,3 \%$ & $8,6 \%$ \\
\hline De toda la vida & $34,1 \%$ & $40,5 \%$ & $37,8 \%$ & $38,3 \%$ & $27,9 \%$ & $34,2 \%$ & $38,9 \%$ & $33,3 \%$ & $36,7 \%$ \\
\hline Por otras personas & $21,5 \%$ & $19,0 \%$ & $20,0 \%$ & $16,8 \%$ & $20,6 \%$ & $22,1 \%$ & $13,7 \%$ & $11,6 \%$ & $18,4 \%$ \\
\hline Señalización carretera & $5,9 \%$ & $4,5 \%$ & $7,8 \%$ & $15,0 \%$ & $5,9 \%$ & $10,1 \%$ & $9,9 \%$ & $15,3 \%$ & $8,8 \%$ \\
\hline Oficinas de turismo & $5,2 \%$ & $3,8 \%$ & $4,1 \%$ & $1,9 \%$ & $5,9 \%$ & $1,5 \%$ & $1,5 \%$ & $1,1 \%$ & $3,1 \%$ \\
\hline Mapas, guías, libros & $6,7 \%$ & $5,5 \%$ & $1,1 \%$ & $12,1 \%$ & $4,4 \%$ & $1,5 \%$ & $5,3 \%$ & $4,2 \%$ & $6,4 \%$ \\
\hline Internet & $4,4 \%$ & $6,2 \%$ & $5,0 \%$ & $5,6 \%$ & $8,8 \%$ & $1,0 \%$ & $4,6 \%$ & $1,6 \%$ & $4,4 \%$ \\
\hline Otros medios & $1,5 \%$ & $1,0 \%$ & $1,6 \%$ & $0,0 \%$ & $2,9 \%$ & $1,5 \%$ & $3,8 \%$ & $2,1 \%$ & $1,7 \%$ \\
\hline Otras medios & $3,0 \%$ & $3,5 \%$ & $3,2 \%$ & $0,9 \%$ & $2,9 \%$ & $1,5 \%$ & $0,8 \%$ & $0,0 \%$ & $2,3 \%$ \\
\hline \multicolumn{10}{|l|}{ Composición del grupo $^{* *}$} \\
\hline $\begin{array}{l}\text { Individual } \\
\text { Pareja }\end{array}$ & $\begin{array}{r}5,2 \% \\
29,7 \%\end{array}$ & $\begin{array}{r}9,0 \% \\
29,8 \%\end{array}$ & $\begin{array}{r}8,0 \% \\
35,5 \%\end{array}$ & $\begin{array}{l}13,1 \% \\
26,2 \%\end{array}$ & $\begin{array}{l}30,9 \% \\
13,2 \%\end{array}$ & $\begin{array}{l}11,6 \% \\
24,1 \%\end{array}$ & $\begin{array}{r}3,8 \% \\
32,1 \%\end{array}$ & $\begin{array}{r}8,5 \% \\
19,0 \%\end{array}$ & $\begin{array}{l}10,1 \% \\
28,3 \%\end{array}$ \\
\hline Familia & $37,0 \%$ & $41,2 \%$ & $31,2 \%$ & $15,0 \%$ & $14,7 \%$ & $30,2 \%$ & $29,8 \%$ & $28,6 \%$ & $31,1 \%$ \\
\hline Amigos & $24,4 \%$ & $18,7 \%$ & $23,2 \%$ & $43,9 \%$ & $39,7 \%$ & $33,6 \%$ & $32,8 \%$ & $40,8 \%$ & $28,6 \%$ \\
\hline Grupos organizados & $3,7 \%$ & $1,3 \%$ & $2,1 \%$ & $1,8 \%$ & $1,5 \%$ & $0,5 \%$ & $1,5 \%$ & $3,1 \%$ & $1,9 \%$ \\
\hline $\begin{array}{l}\text { Media de personas/ } \\
\text { grupo** }\end{array}$ & 5.2 & 3.7 & 3.7 & 3.8 & 2.9 & 3.5 & 3.5 & 5.1 & 3.9 \\
\hline \multicolumn{10}{|l|}{ Alojamiento* } \\
\hline Resido en la zona & $10,4 \%$ & $13,1 \%$ & $6,2 \%$ & $8,4 \%$ & $17,7 \%$ & $21,1 \%$ & $14,5 \%$ & $19,0 \%$ & $13,2 \%$ \\
\hline Sin alojamiento & $13,3 \%$ & $8,7 \%$ & $3,4 \%$ & $5,3 \%$ & $5,9 \%$ & $40,8 \%$ & $24,3 \%$ & $11,1 \%$ & $13,1 \%$ \\
\hline $2^{\mathrm{a}}$ residencia, piso & $20,7 \%$ & $22,5 \%$ & $17,9 \%$ & $3,7 \%$ & $14,7 \%$ & $15,6 \%$ & $17,5 \%$ & $14,8 \%$ & $17,2 \%$ \\
\hline Casa rural & $5,9 \%$ & $9,3 \%$ & $8,7 \%$ & $1,9 \%$ & $4,4 \%$ & $1,5 \%$ & $3,8 \%$ & $5,3 \%$ & $6,3 \%$ \\
\hline Casa amigos/familiares & $5,9 \%$ & $4,5 \%$ & $6,2 \%$ & $3,7 \%$ & $2,9 \%$ & $2,0 \%$ & $3,1 \%$ & $9,0 \%$ & $4,9 \%$ \\
\hline Hotel, hostal & $24,5 \%$ & $19,5 \%$ & $21,9 \%$ & $11,5 \%$ & $13,2 \%$ & $7,0 \%$ & $19,1 \%$ & $20,2 \%$ & $17,9 \%$ \\
\hline Camping & $12,6 \%$ & $16,3 \%$ & $21,3 \%$ & $12,1 \%$ & $19,2 \%$ & $3,5 \%$ & $11,5 \%$ & $3,2 \%$ & $13,6 \%$ \\
\hline Albergue & $3,0 \%$ & $3,8 \%$ & $4,1 \%$ & $5,6 \%$ & $13,2 \%$ & $5,0 \%$ & $3,1 \%$ & $10,1 \%$ & $5,1 \%$ \\
\hline Refugio de montaña & $2,2 \%$ & $1,0 \%$ & $5,0 \%$ & $14,1 \%$ & $4,4 \%$ & $1,0 \%$ & $0,0 \%$ & $0,5 \%$ & $3,1 \%$ \\
\hline Vivac & $0,0 \%$ & $0,3 \%$ & $3,9 \%$ & $27,2 \%$ & $0,0 \%$ & $1,0 \%$ & $2,3 \%$ & $0,5 \%$ & $3,3 \%$ \\
\hline Caravana & $1,5 \%$ & $1,0 \%$ & $1,4 \%$ & $6,5 \%$ & $4,4 \%$ & $1,5 \%$ & $0,8 \%$ & $6,3 \%$ & $2,3 \%$ \\
\hline \multicolumn{10}{|l|}{ Medio de transporte* } \\
\hline Coche particular & $94,1 \%$ & $86,9 \%$ & $77,5 \%$ & $94,4 \%$ & $27,9 \%$ & $95,5 \%$ & $80,8 \%$ & $95,3 \%$ & $84,5 \%$ \\
\hline Taxi & $0,0 \%$ & $1,4 \%$ & $0,9 \%$ & $1,9 \%$ & $0,0 \%$ & $0,5 \%$ & $0,8 \%$ & $2,1 \%$ & $1,1 \%$ \\
\hline Bicicleta montaña & $0,7 \%$ & $1,0 \%$ & $1,4 \%$ & $0,0 \%$ & $67,7 \%$ & $0,5 \%$ & $0,0 \%$ & $0,5 \%$ & $3,8 \%$ \\
\hline Andando & $3,0 \%$ & $10,4 \%$ & $18,1 \%$ & $2,8 \%$ & $2,9 \%$ & $1,5 \%$ & $0,8 \%$ & $1,6 \%$ & $7,9 \%$ \\
\hline Quad-moto & $0,7 \%$ & $0,0 \%$ & $0,5 \%$ & $0,0 \%$ & $0,0 \%$ & $0,5 \%$ & $13,0 \%$ & $0,0 \%$ & $1,3 \%$ \\
\hline Otro & $1,5 \%$ & $0,3 \%$ & $1,6 \%$ & $0,9 \%$ & $1,5 \%$ & $1,5 \%$ & $4,6 \%$ & $0,5 \%$ & $1,4 \%$ \\
\hline \multicolumn{10}{|l|}{ Frecuencia de visitas** } \\
\hline Muy a menudo-vivo zona & $13,3 \%$ & $13,1 \%$ & $9,4 \%$ & $7,5 \%$ & $16,2 \%$ & $13,6 \%$ & $18,2 \%$ & $15,3 \%$ & $13,3 \%$ \\
\hline Primera vez & $39,2 \%$ & $35,0 \%$ & $38,8 \%$ & $41,1 \%$ & $35,2 \%$ & $25,1 \%$ & $38,2 \%$ & $33,3 \%$ & $34,9 \%$ \\
\hline Entre 2 y 3 veces & $10,4 \%$ & $20,4 \%$ & $18,1 \%$ & $16,8 \%$ & $4,4 \%$ & $9,0 \%$ & $14,5 \%$ & $9,5 \%$ & $14,8 \%$ \\
\hline Entre 4 y 6 veces & $21,5 \%$ & $16,6 \%$ & $19,5 \%$ & $26,2 \%$ & $32,4 \%$ & $26,6 \%$ & $17,6 \%$ & $13,8 \%$ & $19,8 \%$ \\
\hline Más de 7 veces & $15,6 \%$ & $14,9 \%$ & $14,2 \%$ & $8,4 \%$ & $11,8 \%$ & $25,7 \%$ & $11,5 \%$ & $28,1 \%$ & $17,2 \%$ \\
\hline $\begin{array}{l}\text { Duración de la visita } \\
\text { zona* }\end{array}$ & 3,3 días & 4,8 días & 5,5 días & 3,2 días & 3,7 días & 2,2 días & 2,7 días & 2,2 días & 3,9 días \\
\hline \multicolumn{10}{|l|}{ Duración de visita } \\
\hline 1/2 día & $\begin{array}{l}83,0 \% \\
14,7 \%\end{array}$ & $\begin{array}{l}86,5 \% \\
11,4 \%\end{array}$ & $\begin{array}{l}69,1 \% \\
28,8 \%\end{array}$ & $\begin{array}{l}15,8 \% \\
45,9 \%\end{array}$ & $\begin{array}{l}6 /, 1 \% \\
32,3 \%\end{array}$ & $\begin{array}{l}13,4 \% \\
26,1 \%\end{array}$ & $\begin{array}{l}85,6 \% \\
13,6 \%\end{array}$ & $\begin{array}{l}70,3 \% \\
20,2 \%\end{array}$ & $\begin{array}{l}71,8 \% \\
22,3 \%\end{array}$ \\
\hline 1 día & $2,3 \%$ & $1,8 \%$ & $1,1 \%$ & $37,4 \%$ & $0,0 \%$ & $0,5 \%$ & $0,8 \%$ & $9,5 \%$ & $4,8 \%$ \\
\hline $\begin{array}{l}\text { Entre } 2 \text { y } 3 \text { dias } \\
\text { Más de } 3 \text { días }\end{array}$ & $0,0 \%$ & $0,3 \%$ & $0,7 \%$ & $0,9 \%$ & $0,0 \%$ & $0,0 \%$ & $0,0 \%$ & $0,0 \%$ & $0,3 \%$ \\
\hline Duración media visita** & 4,3 horas & 4,2 horas & 6,0 horas & 25,1 horas & 4,8 horas & 5,4 horas & 3,8 horas & 9,0 horas & 6,95 horas \\
\hline \multicolumn{10}{|l|}{ Motivos de la visita* } \\
\hline Estar cerca naturaleza & $27,5 \%$ & $31,0 \%$ & $30,2 \%$ & $27,70 \%$ & $22,8 \%$ & $22,3 \%$ & $32,3 \%$ & $24,7 \%$ & $28,1 \%$ \\
\hline Practicar actividad física & $11,4 \%$ & $11,8 \%$ & $20,1 \%$ & $21,40 \%$ & $36,4 \%$ & $6,0 \%$ & $6,6 \%$ & $34,9 \%$ & $17,9 \%$ \\
\hline Visitar itinerario particular & $6,5 \%$ & $6,6 \%$ & $11,5 \%$ & $15,70 \%$ & $6,8 \%$ & $3,7 \%$ & $12,3 \%$ & $4,2 \%$ & $8,5 \%$ \\
\hline Estar con familia/amigos & $21,8 \%$ & $15,4 \%$ & $9,9 \%$ & $9,80 \%$ & $8,0 \%$ & $9,7 \%$ & $12,3 \%$ & $17,1 \%$ & $12,8 \%$ \\
\hline Conocer nuevos sitios & $9,8 \%$ & $9,1 \%$ & $9,5 \%$ & $9,80 \%$ & $7,5 \%$ & $1,6 \%$ & $11,6 \%$ & $9,6 \%$ & $8,7 \%$ \\
\hline Relajarme y desconectar & $17,8 \%$ & $19,7 \%$ & $14,0 \%$ & $12,20 \%$ & $14,2 \%$ & $10,8 \%$ & $18,3 \%$ & $7,3 \%$ & $14,4 \%$ \\
\hline Recolectar setas & $0,9 \%$ & $2,9 \%$ & $2,2 \%$ & $1,70 \%$ & $2,5 \%$ & $43,8 \%$ & $3,3 \%$ & $0,4 \%$ & $6,3 \%$ \\
\hline Por trabajo & $0,3 \%$ & $0,3 \%$ & $0,4 \%$ & $0,00 \%$ & $0,6 \%$ & $0,5 \%$ & $0,0 \%$ & $0,9 \%$ & $0,6 \%$ \\
\hline Otras razones & $4,0 \%$ & $3,2 \%$ & $2,2 \%$ & $1,70 \%$ & $1,2 \%$ & $1,6 \%$ & $3,3 \%$ & $0,9 \%$ & $2,7 \%$ \\
\hline
\end{tabular}




\begin{tabular}{|c|c|c|c|c|c|c|c|c|c|}
\hline Variables & $\begin{array}{c}\text { Paseantes } \\
n=135\end{array}$ & $\begin{array}{c}\text { Excursionistas } \\
\mathbf{n}=\mathbf{2 8 9}\end{array}$ & $\begin{array}{c}\text { Senderistas } \\
n=436\end{array}$ & $\begin{array}{c}\text { Montañistas } \\
\mathbf{n}=\mathbf{1 0 7}\end{array}$ & $\begin{array}{c}\begin{array}{c}\text { Ciclistas } \\
n=68\end{array} \\
\text {. }\end{array}$ & $\begin{array}{c}\text { Recolectores } \\
\text { de setas } \\
\mathbf{n}=199\end{array}$ & $\begin{array}{c}\text { Motoristas } \\
n=131\end{array}$ & $\begin{array}{c}\text { Esquiadores } \\
\mathbf{y} \text { otras } \\
\mathbf{n}=189\end{array}$ & $\begin{array}{c}\text { Total } \\
\text { Muestra }\end{array}$ \\
\hline $\begin{array}{l}\text { Tipología itinerario** } \\
\text { Clásico } \\
\text { No clásico } \\
\text { No visita el parque }\end{array}$ & $\begin{array}{r}46,7 \% \\
5,9 \% \\
47,4 \%\end{array}$ & $\begin{array}{r}81,0 \% \\
4,5 \% \\
14,5 \%\end{array}$ & $\begin{array}{r}84,4 \% \\
4,1 \% \\
11,5 \%\end{array}$ & $\begin{array}{r}90,7 \% \\
8,4 \% \\
0,9 \%\end{array}$ & $\begin{array}{r}77,9 \% \\
16,2 \% \\
5,9 \%\end{array}$ & $\begin{array}{r}91,5 \% \\
2,5 \% \\
6,0 \%\end{array}$ & $\begin{array}{r}84,7 \% \\
4,6 \% \\
10,7 \%\end{array}$ & $\begin{array}{r}61,9 \% \\
0,5 \% \\
37,6 \%\end{array}$ & $\begin{array}{r}78,3 \% \\
4,7 \% \\
17,0 \%\end{array}$ \\
\hline $\begin{array}{l}\text { Visita a otros lugares** } \\
\text { Sí } \\
\text { No } \\
\text { No lo sé }\end{array}$ & $\begin{array}{r}40,0 \% \\
54,1 \% \\
5,9 \%\end{array}$ & $\begin{array}{r}49,1 \% \\
43,9 \% \\
6,9 \%\end{array}$ & $\begin{array}{r}47,9 \% \\
46,8 \% \\
5,3 \%\end{array}$ & $\begin{array}{r}23,4 \% \\
72,9 \% \\
3,7 \%\end{array}$ & $\begin{array}{r}55,9 \% \\
38,2 \% \\
5,9 \%\end{array}$ & $\begin{array}{r}35,7 \% \\
55,8 \% \\
8,5 \%\end{array}$ & $\begin{array}{r}32,8 \% \\
60,3 \% \\
6,9 \%\end{array}$ & $\begin{array}{r}31,2 \% \\
66,7 \% \\
2,1 \%\end{array}$ & $\begin{array}{r}41,6 \% \\
52,9 \% \\
5,6 \%\end{array}$ \\
\hline $\begin{array}{l}\text { Meses del año }{ }^{* * *} \\
\text { Enero } \\
\text { Febrero } \\
\text { Marzo } \\
\text { Abril } \\
\text { Mayo } \\
\text { Junio } \\
\text { Julio } \\
\text { Agosto } \\
\text { Septiembre } \\
\text { Octubre } \\
\text { Diciembre }\end{array}$ & $\begin{array}{r}14,8 \% \\
4,4 \% \\
7,4 \% \\
11,1 \% \\
7,4 \% \\
12,6 \% \\
3,7 \% \\
21,5 \% \\
8,1 \% \\
4,4 \% \\
4,4 \%\end{array}$ & $\begin{array}{r}2,4 \% \\
1,7 \% \\
0,3 \% \\
10,0 \% \\
6,2 \% \\
9,0 \% \\
14,4 \% \\
37,7 \% \\
12,8 \% \\
3,1 \% \\
2,4 \%\end{array}$ & $\begin{array}{r}1,4 \% \\
02 \% \\
0,5 \% \\
9,4 \% \\
4,1 \% \\
9,4 \% \\
14,7 \% \\
42,0 \% \\
11,5 \% \\
6,0 \% \\
0,9 \%\end{array}$ & $\begin{array}{r}0,9 \% \\
0,9 \% \\
0,0 \% \\
1,9 \% \\
0,9 \% \\
10,3 \% \\
18,7 \% \\
35,5 \% \\
26,2 \% \\
4,7 \% \\
0,0 \%\end{array}$ & $\begin{array}{r}0,0 \% \\
0,0 \% \\
0,0 \% \\
8,8 \% \\
5,9 \% \\
7,4 \% \\
20,6 \% \\
45,6 \% \\
7,4 \% \\
4,4 \% \\
0,0 \%\end{array}$ & $\begin{array}{r}0,0 \% \\
0,0 \% \\
0,0 \% \\
0,5 \% \\
8,5 \% \\
10,1 \% \\
8,5 \% \\
19,1 \% \\
37,7 \% \\
15,6 \% \\
0,0 \%\end{array}$ & $\begin{array}{r}0,8 \% \\
0,0 \% \\
0,8 \% \\
15,3 \% \\
5,3 \% \\
6,1 \% \\
16,0 \% \\
35,1 \% \\
9,9 \% \\
9,9 \% \\
0,8 \%\end{array}$ & $\begin{array}{r}18,5 \% \\
26,5 \% \\
23,8 \% \\
0,0 \% \\
0,0 \% \\
0,0 \% \\
0,0 \% \\
0,0 \% \\
0,0 \% \\
0,0 \% \\
30,2 \%\end{array}$ & $\begin{array}{r}4,4 \% \\
4,0 \% \\
3,7 \% \\
7,3 \% \\
5,0 \% \\
8,1 \% \\
12,1 \% \\
30,6 \% \\
14,0 \% \\
5,9 \% \\
4,8 \%\end{array}$ \\
\hline
\end{tabular}

$\mathrm{n}=1554$

*Diferencias entre grupos con un nivel de significación de 0.05

** Diferencias entre grupos con un nivel de significación de 0.01 $n=1554$

* Differences between groups are significant at the 0.05 level

** Differences between groups are significant at the 0.01 level

cios que más les gustaría encontrar en un futuro en el parque son: los itinerarios interpretativos $(20,2 \%)$, los puntos de información $(18,3 \%)$ y los servicios de vigilancia y control ambiental $(16,4 \%)$. Finalmente, la mayor parte de los visitantes encuestados manifiesta que no es necesario instaurar nuevas regulaciones de uso: $52,4 \%$ en el caso de la recolección de setas y $41,8 \%$ en el caso de la circulación motorizada (Tabla 5). Téngase en cuenta que en esta última pregunta, un $25,8 \%$ de los visitantes no opinaron por el hecho de no haber visitado zonas con necesidades de regulación.

\subsection{Perfil de los grupos de visitantes}

\section{Paseantes}

Este es el grupo de visitantes que no realiza ningún tipo de actividad específica durante su visita al Parque Natural. Suelen permanecer próximos a los puntos de acceso, ya sea observando la naturaleza, comiendo o, simplemente, haciendo vida social. Está constituido por 135 sujetos $(8,4 \%)$ y es uno de los grupos de visitantes con mayor presencia de mujeres $(41,5 \%)$. Acceden al parque en grupo con uno de los ratios de visitantes/grupo más elevados (5,2 personas/ grupo). Podría decirse que destacan por su perfil pasivo. Son de los que más utilizan el coche para acceder al parque $(94,1 \%)$, y una vez en el mismo, no se adentran en su territorio (el 47,4\% no visitan el territorio del Parque Natural como tal, sino que se quedan próximos a los accesos). Así mismo, son los visitantes que, respecto a la media, más utilizan los hoteles para alojarse $(24,5 \%) \mathrm{y}$, curiosamente, son el tercer grupo que menos conocimientos tiene sobre el estatus de protección del parque (el 21,5\% no sabe que el
PNAP es un espacio natural protegido). Suelen visitar el parque entrando por los puntos de entrada más habituales, es decir, Tavascan (30,5\%) y Sant Joan de l'Erm (23,8\%) figura 2 - y no permanecen en el mismo más de medio día $(86,5 \%)$. Visitan el parque sobre todo durante los meses de Enero (14,8\%) y Agosto (21,5\%). Estar cerca de la naturaleza $(27,5 \%)$, estar con familia/amigos $(21,8 \%)$ y relajarse $y$ desconectar, $(17,8 \%)$ son, para este grupo, las tres motivaciones principales de concurrencia al Parque. Al igual que el resto de visitantes, valoran positivamente la mayor parte los aspectos de gestión del parque y no son partidarios de regular ni la recolección de setas $(50,4 \%)$, ni la circulación de vehículos motorizados $(48,1 \%)$. Ver tablas 3, 4 y 5 .

\section{Excursionistas}

Este grupo está formado por un total de 289 sujetos $(18,1 \%)$, el segundo grupo más numeroso. Durante su visita realizan caminatas no superiores a una hora y se caracterizan por residir de forma habitual en la provincia de Barcelona $(61,9 \%)$. Es el grupo de visitantes que más utiliza la segunda residencia como alojamiento durante su visita $(22,5 \%)$, visitan el parque por primera vez $(35 \%)$ o entre dos y tres veces $(20,4 \%)$, en familia (41,2\%), durante el mes de Agosto (42,0\%) y lo suelen visitar por el acceso de Tavascan $(25,1 \%)$. Así mismo, y al igual que el conjunto de visitantes, suelen visitar el parque durante medio día $(69,1 \%)$, en familia $(41,2 \%)$, frecuentar de forma mayoritaria itinerarios de tipo clásicos $(81,0 \%)$ y elegir como principales motivaciones de visita: Estar cerca de la naturaleza $(31,0 \%)$, relajarse y desconectar, $(19,7 \%)$ y estar con familia/amigos 
Tabla 5. Variables que mejor definen otros aspectos relativos a las opiniones, percepciones y sugerencias del visitante (ver texto para más detalles). Los valores son porcentajes del número de sujetos por cada grupo.

Table 5. Variables that best define other aspects related to the visitors'opinions, perceptions and suggestions (see text for more details). Values are percentages of the number of subjects for each group

\begin{tabular}{|c|c|c|c|c|c|c|c|c|c|}
\hline Variables & $\begin{array}{l}\text { Paseantes } \\
\mathrm{n}=135\end{array}$ & $\begin{array}{c}\text { Excursionistas } \\
\mathbf{n}=\mathbf{2 8 9}\end{array}$ & $\begin{array}{c}\text { Senderistas } \\
n=436\end{array}$ & $\begin{array}{c}\text { Montañistas } \\
\mathbf{n}=\mathbf{1 0 7}\end{array}$ & $\begin{array}{c}\text { Ciclistas } \\
n=68\end{array}$ & $\begin{array}{c}\text { Recolectores } \\
\text { de setas } \\
n=199\end{array}$ & $\begin{array}{c}\text { Motoristas } \\
\quad n=131\end{array}$ & $\begin{array}{c}\text { Esquiadores } \\
\mathbf{y} \text { otras } \\
\mathbf{n}=\mathbf{1 8 9}\end{array}$ & $\begin{array}{c}\text { Total } \\
\text { Muestra }\end{array}$ \\
\hline $\begin{array}{l}\text { Experiencia de conflicto* } \\
\text { Ningún problema } \\
\text { Con otros visitantes } \\
\text { Con otras practicas } \\
\text { Con otros usos y otros }\end{array}$ & $\begin{array}{r}96,3 \% \\
1,5 \% \\
0,7 \% \\
1,5 \%\end{array}$ & $\begin{array}{r}93,4 \% \\
0,7 \% \\
5,2 \% \\
0,6 \%\end{array}$ & $\begin{array}{r}94,7 \% \\
1,8 \% \\
2,5 \% \\
0,9 \%\end{array}$ & $\begin{array}{r}91,6 \% \\
2,8 \% \\
0,9 \% \\
4,6 \%\end{array}$ & $\begin{array}{r}88,2 \% \\
0,0 \% \\
10,3 \% \\
1,5 \%\end{array}$ & $\begin{array}{r}95,0 \% \\
0,5 \% \\
3,5 \% \\
1,0 \%\end{array}$ & $\begin{array}{r}93,1 \% \\
2,3 \% \\
4,6 \% \\
0,0 \%\end{array}$ & $\begin{array}{r}97,9 \% \\
0,0 \% \\
2,1 \% \\
0,0 \%\end{array}$ & $\begin{array}{r}94,3 \% \\
1,3 \% \\
3,3 \% \\
1,1 \%\end{array}$ \\
\hline $\begin{array}{l}\text { Masificación* } \\
\text { Excesiva } \\
\text { Aceptable } \\
\text { Poca gente }\end{array}$ & $\begin{array}{l}11,1 \% \\
60,0 \% \\
28,9 \%\end{array}$ & $\begin{array}{r}4,5 \% \\
65,7 \% \\
29,8 \%\end{array}$ & $\begin{array}{r}3,9 \% \\
61,0 \% \\
35,1 \%\end{array}$ & $\begin{array}{r}9,3 \% \\
68,3 \% \\
22,4 \%\end{array}$ & $\begin{array}{l}10,3 \% \\
75,0 \% \\
14,7 \%\end{array}$ & $\begin{array}{l}15,1 \% \\
65,8 \% \\
19,1 \%\end{array}$ & $\begin{array}{r}4,6 \% \\
67,2 \% \\
28,2 \%\end{array}$ & $\begin{array}{r}1,6 \% \\
74,1 \% \\
24,3 \%\end{array}$ & $\begin{array}{r}6,7 \% \\
65,5 \% \\
27,8 \%\end{array}$ \\
\hline $\begin{array}{l}\text { Señalización** } \\
\text { No corresponde } \\
\text { Muy correcta } \\
\text { Correcta } \\
\text { Regular } \\
\text { Incorrecta }\end{array}$ & $\begin{array}{r}21,5 \% \\
17,0 \% \\
48,2 \% \\
9,6 \% \\
3,7 \%\end{array}$ & $\begin{array}{r}7,6 \% \\
26,6 \% \\
49,5 \% \\
13,5 \% \\
2,8 \%\end{array}$ & $\begin{array}{r}3,0 \% \\
23,2 \% \\
47,1 \% \\
20,0 \% \\
6,7 \%\end{array}$ & $\begin{array}{r}2,8 \% \\
15,0 \% \\
64,4 \% \\
15,0 \% \\
2,8 \%\end{array}$ & $\begin{array}{r}2,9 \% \\
20,6 \% \\
51,5 \% \\
22,1 \% \\
2,9 \%\end{array}$ & $\begin{array}{r}11,6 \% \\
12,1 \% \\
63,2 \% \\
11,1 \% \\
2,0 \%\end{array}$ & $\begin{array}{r}6,1 \% \\
21,4 \% \\
55,7 \% \\
13,0 \% \\
3,8 \%\end{array}$ & $\begin{array}{r}23,3 \% \\
5,3 \% \\
61,9 \% \\
7,9 \% \\
1,6 \%\end{array}$ & $\begin{array}{r}9,3 \% \\
18,9 \% \\
53,6 \% \\
14,3 \% \\
3,9 \%\end{array}$ \\
\hline $\begin{array}{l}\text { Infraestructuras** } \\
\text { No corresponde } \\
\text { Muy correcta } \\
\text { Correcta } \\
\text { Regular } \\
\text { Incorrecta }\end{array}$ & $\begin{array}{r}18,5 \% \\
14,1 \% \\
63,0 \% \\
3,7 \% \\
0,7 \%\end{array}$ & $\begin{array}{r}18,0 \% \\
22,8 \% \\
51,6 \% \\
5,9 \% \\
1,7 \%\end{array}$ & $\begin{array}{r}20,9 \% \\
20,2 \% \\
45,8 \% \\
10,1 \% \\
3,0 \%\end{array}$ & $\begin{array}{r}15,0 \% \\
9,3 \% \\
57,0 \% \\
15,0 \% \\
3,7 \%\end{array}$ & $\begin{array}{r}25,0 \% \\
25,0 \% \\
45,6 \% \\
1,5 \% \\
2,9 \%\end{array}$ & $\begin{array}{r}17,1 \% \\
21,6 \% \\
55,8 \% \\
5,5 \% \\
0,0 \%\end{array}$ & $\begin{array}{r}32,8 \% \\
14,5 \% \\
48,9 \% \\
2,3 \% \\
1,5 \%\end{array}$ & $\begin{array}{r}6,3 \% \\
14,8 \% \\
75,2 \% \\
3,7 \% \\
0,0 \%\end{array}$ & $\begin{array}{r}18,6 \% \\
18,9 \% \\
53,8 \% \\
6,9 \% \\
1,8 \%\end{array}$ \\
\hline $\begin{array}{l}\text { Folletos, mapas** } \\
\text { No corresponde } \\
\text { Muy correcta } \\
\text { Correcta } \\
\text { Regular } \\
\text { Incorrecta }\end{array}$ & $\begin{array}{r}51,8 \% \\
16,3 \% \\
27,4 \% \\
3,0 \% \\
1,5 \%\end{array}$ & \begin{tabular}{r|}
$43,0 \%$ \\
$19,0 \%$ \\
$32,5 \%$ \\
$5,2 \%$ \\
$0,3 \%$
\end{tabular} & $\begin{array}{r}43,1 \% \\
21,3 \% \\
29,4 \% \\
4,4 \% \\
1,8 \%\end{array}$ & $\begin{array}{r}68,2 \% \\
9,3 \% \\
20,6 \% \\
1,9 \% \\
0,0 \%\end{array}$ & $\begin{array}{r}41,2 \% \\
25,0 \% \\
30,9 \% \\
2,9 \% \\
0,0 \%\end{array}$ & $\begin{array}{r}65,9 \% \\
5,5 \% \\
26,6 \% \\
2,0 \% \\
0,0 \%\end{array}$ & $\begin{array}{r}61,9 \% \\
13,7 \% \\
22,1 \% \\
2,3 \% \\
0,0 \%\end{array}$ & $\begin{array}{r}60,8 \% \\
10,1 \% \\
27,0 \% \\
1,6 \% \\
0,5 \%\end{array}$ & $\begin{array}{r}52,0 \% \\
15,8 \% \\
27,9 \% \\
3,5 \% \\
0,8 \%\end{array}$ \\
\hline $\begin{array}{l}\text { Limpieza* } \\
\text { No corresponde } \\
\text { Muy correcta } \\
\text { Correcta } \\
\text { Regular } \\
\text { Incorrecta }\end{array}$ & $\begin{array}{r}5,2 \% \\
24,4 \% \\
63,0 \% \\
5,9 \% \\
1,5 \%\end{array}$ & \begin{tabular}{r|}
$2,8 \%$ \\
$37,4 \%$ \\
$50,1 \%$ \\
$8,7 \%$ \\
$1,0 \%$
\end{tabular} & $\begin{array}{r}0,2 \% \\
41,5 \% \\
51,1 \% \\
6,7 \% \\
0,5 \%\end{array}$ & $\begin{array}{r}0,0 \% \\
29,0 \% \\
59,8 \% \\
11,2 \% \\
0,0 \%\end{array}$ & $\begin{array}{r}0,0 \% \\
52,9 \% \\
35,4 \% \\
8,8 \% \\
2,9 \%\end{array}$ & $\begin{array}{r}2,5 \% \\
15,1 \% \\
59,3 \% \\
18,1 \% \\
5,0 \%\end{array}$ & $\begin{array}{r}0,8 \% \\
43,5 \% \\
50,4 \% \\
5,3 \% \\
0,0 \%\end{array}$ & $\begin{array}{r}7,4 \% \\
18,0 \% \\
71,5 \% \\
2,6 \% \\
0,5 \%\end{array}$ & $\begin{array}{r}2,3 \% \\
32,6 \% \\
55,3 \% \\
8,2 \% \\
1,6 \%\end{array}$ \\
\hline $\begin{array}{l}\text { Demanda instalaciones* } \\
\text { Miradores panorámicos } \\
\text { Itinerarios interpretativos } \\
\text { Puntos de información } \\
\text { Servicio de guías } \\
\text { Servicio de conservación } \\
\text { Otras instalaciones } \\
\text { Todas instalaciones } \\
\text { No selecciona ninguno }\end{array}$ & $\begin{array}{r}15,2 \% \\
18,8 \% \\
18,4 \% \\
9,0 \% \\
16,1 \% \\
3,1 \% \\
1,8 \% \\
17,5 \%\end{array}$ & $\begin{array}{r}15,7 \% \\
23,6 \% \\
18,4 \% \\
9,7 \% \\
14,7 \% \\
5,4 \% \\
1,4 \% \\
11,2 \%\end{array}$ & $\begin{array}{r}14,0 \% \\
24,7 \% \\
18,5 \% \\
7,7 \% \\
16,4 \% \\
7,7 \% \\
0,6 \% \\
10,5 \%\end{array}$ & $\begin{array}{r}7,3 \% \\
17,5 \% \\
13,9 \% \\
5,8 \% \\
11,7 \% \\
14,6 \% \\
0,7 \% \\
28,5 \%\end{array}$ & $\begin{array}{r}10,0 \% \\
24,0 \% \\
16,0 \% \\
13,0 \% \\
14,0 \% \\
6,0 \% \\
2,0 \% \\
15,0 \%\end{array}$ & $\begin{array}{r}13,5 \% \\
13,1 \% \\
20,9 \% \\
5,7 \% \\
25,0 \% \\
3,3 \% \\
0,0 \% \\
18,4 \%\end{array}$ & $\begin{array}{r}18,5 \% \\
13,5 \% \\
20,2 \% \\
8,4 \% \\
12,9 \% \\
6,2 \% \\
0,0 \% \\
20,2 \%\end{array}$ & $\begin{array}{r}9,4 \% \\
17,0 \% \\
17,3 \% \\
17,3 \% \\
16,1 \% \\
4,0 \% \\
0,0 \% \\
18,8 \%\end{array}$ & $\begin{array}{r}13,5 \% \\
20,2 \% \\
18,3 \% \\
9,5 \% \\
16,4 \% \\
6,1 \% \\
0,7 \% \\
15,2 \%\end{array}$ \\
\hline $\begin{array}{l}\text { Regulación de recolección } \\
\text { setas** } \\
\text { No responde } \\
\text { No hace falta regular } \\
\mathrm{Si} \text {, límite diario de setas } \\
\mathrm{Si} \text {, cobrar tasa } \\
\text { Educar para un buen uso } \\
\text { Sí, otras opciones } \\
\text { No tengo opinión } \\
\text { Si, las dos (límite y tasa) }\end{array}$ & $\begin{array}{r}13,3 \% \\
50,4 \% \\
13,3 \% \\
7,4 \% \\
3,0 \% \\
2,2 \% \\
5,2 \% \\
5,2 \%\end{array}$ & $\begin{array}{r}19,0 \% \\
52,6 \% \\
11,1 \% \\
5,9 \% \\
2,4 \% \\
1,0 \% \\
2,1 \% \\
5,9 \%\end{array}$ & $\begin{array}{r}19,3 \% \\
51,1 \% \\
10,6 \% \\
6,7 \% \\
2,5 \% \\
0,9 \% \\
4,8 \% \\
4,1 \%\end{array}$ & $\begin{array}{r}26,2 \% \\
45,8 \% \\
8,4 \% \\
5,6 \% \\
4,70 \% \\
0,0 \% \\
8,4 \% \\
0,9 \%\end{array}$ & $\begin{array}{r}5,9 \% \\
51,5 \% \\
13,1 \% \\
11,8 \% \\
7,4 \% \\
2,9 \% \\
1,5 \% \\
5,9 \%\end{array}$ & $\begin{array}{r}1,5 \% \\
70,9 \% \\
10,1 \% \\
5,0 \% \\
0,8 \% \\
0,8 \% \\
3,1 \% \\
3,1 \%\end{array}$ & $\begin{array}{r}16,8 \% \\
62,6 \% \\
7,6 \% \\
3,2 \% \\
3,2 \% \\
2,1 \% \\
4,2 \% \\
2,1 \%\end{array}$ & $\begin{array}{r}37,6 \% \\
36,0 \% \\
11,6 \% \\
8,7 \% \\
2,0 \% \\
4,3 \% \\
0,0 \% \\
13,0 \%\end{array}$ & $\begin{array}{r}18,4 \% \\
52,4 \% \\
10,5 \% \\
6,2 \% \\
3,1 \% \\
1,6 \% \\
3,5 \% \\
4,3 \%\end{array}$ \\
\hline $\begin{array}{l}\text { Regulación de circulación } \\
\text { motorizada** } \\
\text { No responde } \\
\text { No hace falta } \\
\mathrm{Si} \text {, hace falta hacer algo } \\
\text { Hacer cumplir la normativa } \\
\mathrm{Si} \text {, dependiendo de época } \\
\text { Restringir uso } \\
\text { Cerrar circulación } \\
\text { Cerrar totalmente } \\
\text { Depende del lugar }\end{array}$ & $\begin{array}{r}15,6 \% \\
48,1 \% \\
5,9 \% \\
3,0 \% \\
7,4 \% \\
6,7 \% \\
4,4 \% \\
2,2 \% \\
6,7 \%\end{array}$ & \begin{tabular}{r|}
$25,6 \%$ \\
$48,1 \%$ \\
$4,2 \%$ \\
$8,3 \%$ \\
$2,8 \%$ \\
$2,8 \%$ \\
$3,5 \%$ \\
$3,5 \%$ \\
$1,4 \%$
\end{tabular} & $\begin{array}{r}31,4 \% \\
39,4 \% \\
6,4 \% \\
8,5 \% \\
4,8 \% \\
2,1 \% \\
2,1 \% \\
2,8 \% \\
2,5 \%\end{array}$ & $\begin{array}{r}53,9 \% \\
35,5 \% \\
2,8 \% \\
2,8 \% \\
7,5 \% \\
1,9 \% \\
2,8 \% \\
2,8 \% \\
0,0 \%\end{array}$ & $\begin{array}{r}13,2 \% \\
26,5 \% \\
4,4 \% \\
19,1 \% \\
8,8 \% \\
7,4 \% \\
2,9 \% \\
11,8 \% \\
5,9 \%\end{array}$ & $\begin{array}{r}19,1 \% \\
50,8 \% \\
3,5 \% \\
12,6 \% \\
2,5 \% \\
3,0 \% \\
3,0 \% \\
2,0 \% \\
3,5 \%\end{array}$ & $\begin{array}{r}18,3 \% \\
52,7 \% \\
4,6 \% \\
12,2 \% \\
3,8 \% \\
1,5 \% \\
0,8 \% \\
0,0 \% \\
6,1 \%\end{array}$ & $\begin{array}{r}27,0 \% \\
26,5 \% \\
11,6 \% \\
6,3 \% \\
12,2 \% \\
2,1 \% \\
3,7 \% \\
5,9 \% \\
4,8 \%\end{array}$ & $\begin{array}{r}25,8 \% \\
41,8 \% \\
5,7 \% \\
8,8 \% \\
5,5 \% \\
3,0 \% \\
2,9 \% \\
3,3 \% \\
3,4 \%\end{array}$ \\
\hline
\end{tabular}




\begin{tabular}{|c|c|c|c|c|c|c|c|c|c|}
\hline Variables & $\begin{array}{l}\text { Paseantes } \\
n=135\end{array}$ & $\begin{array}{c}\text { Excursionistas } \\
\mathbf{n}=\mathbf{2 8 9}\end{array}$ & $\begin{array}{c}\text { Senderistas } \\
\quad n=436\end{array}$ & $\begin{array}{c}\text { Montañistas } \\
\mathbf{n}=\mathbf{1 0 7}\end{array}$ & $\begin{array}{c}\text { Ciclistas } \\
n=68\end{array}$ & $\begin{array}{c}\text { Recolectores } \\
\text { de setas } \\
n=199\end{array}$ & $\begin{array}{c}\text { Motoristas } \\
n=131\end{array}$ & $\begin{array}{c}\text { Esquiadores } \\
\text { y otras } \\
\mathbf{n}=189\end{array}$ & $\begin{array}{c}\text { Total } \\
\text { Muestra }\end{array}$ \\
\hline Observaciones de mejora* & & & & & & & & & \\
\hline No realiza & $68,1 \%$ & $59,2 \%$ & $51,1 \%$ & $43,9 \%$ & $50,0 \%$ & $64,8 \%$ & $60,3 \%$ & $63,, 5 \%$ & $57,3 \%$ \\
\hline Mejorar señalización & $3,7 \%$ & $9,3 \%$ & $18,1 \%$ & $15,0 \%$ & $11,8 \%$ & $3,5 \%$ & $8,1 \%$ & $6,3 \%$ & $10,7 \%$ \\
\hline Mejorar pistas & $3,0 \%$ & $2,4 \%$ & $5,5 \%$ & $10,3 \%$ & $5,9 \%$ & $8,0 \%$ & $12,2 \%$ & $6,3 \%$ & $6,2 \%$ \\
\hline Mejora equipamientos & $3,7 \%$ & $3,1 \%$ & $2,3 \%$ & $3,7 \%$ & $4,4 \%$ & $2,0 \%$ & $3,1 \%$ & $0,5 \%$ & $2,6 \%$ \\
\hline Mejora Servicios & $5,2 \%$ & $5,2 \%$ & $3,9 \%$ & $0,9 \%$ & $4,4 \%$ & $2,5 \%$ & $3,1 \%$ & $5,3 \%$ & $3,9 \%$ \\
\hline Más limpieza & $3,0 \%$ & $2,10 \%$ & $2,1 \%$ & $1,9 \%$ & $0,0 \%$ & $5,0 \%$ & $0,0 \%$ & $2,1 \%$ & $2,4 \%$ \\
\hline Otras quejas puntuales & $1,5 \%$ & $1,0 \%$ & $1,1 \%$ & $0,0 \%$ & $0,0 \%$ & $0,5 \%$ & $1,5 \%$ & $2,6 \%$ & $1,1 \%$ \\
\hline Controlar la masificación & $3,7 \%$ & $4,5 \%$ & $5,0 \%$ & $10,3 \%$ & $13,2 \%$ & $4,0 \%$ & $6,1 \%$ & $4,8 \%$ & $5,8 \%$ \\
\hline Quejas sobre pernoctación & $0,0 \%$ & $0,7 \%$ & $1,6 \%$ & $7,5 \%$ & $1,5 \%$ & $0,5 \%$ & $0,8 \%$ & $0,0 \%$ & $1,3 \%$ \\
\hline Todo ok & $4,4 \%$ & $7,3 \%$ & $4,8 \%$ & $5,6 \%$ & $4,4 \%$ & $3,0 \%$ & $3,1 \%$ & $6,9 \%$ & $5,1 \%$ \\
\hline Horarios de puntos inf. & $3,7 \%$ & $5,2 \%$ & $3,7 \%$ & $0,9 \%$ & $4,4 \%$ & $6,0 \%$ & $0,8 \%$ & $1,6 \%$ & $3,8 \%$ \\
\hline
\end{tabular}

$\mathrm{n}=1554$

*Diferencias entre grupos con un nivel de significación de 0.05

$* *$ Diferencias entre grupos con un nivel de significación de 0.01 $n=1554$

* Differences between groups are significant at the 0.05 level

** Differences between groups are significant at the 0.01 level

$(15,4)$. Conocen la zona de toda la vida $(40,5 \%)$, visitan otras áreas del parque durante su estancia en la zona $(49,1 \%) \mathrm{y}$, a la hora de valorar los servicios a instaurar, priorizan los de tipo interpretativos (itinerarios interpretativos -23,6\%-, miradores panorámicos $-15,5 \%-$, y puntos de información -18,4\%-) al tiempo que se posicionan en contra de la regulación del acceso motorizado $(48,1 \%)$ y la recolección de setas $(52,6 \%)$. Ver tablas $3,4,5$ y figura 2 .

\section{Senderistas}

Es el grupo más numeroso de visitantes, con un total de 436 senderistas $(27,3 \%)$, procedentes principalmente del área de Barcelona (58,7\%), así como de otras provincias de España (12,8\%). Es el grupo con mayor nivel de formación universitaria $(58,0 \%)$, aunque con un nivel de conocimiento sobre el estatus de protección próximo a la media del conjunto de visitantes (el 85,8\% sí conoce su estatus). Visitan el parque generalmente en pareja (35,5\%) y son uno de los grupos, junto a los montañistas, que más impacto económico generan en la zona a la hora de alojarse (más del 61\% utiliza un alojamiento de pago), siendo los hoteles y campings los alojamientos más utilizados (21,9\% y $21,3 \%$, respectivamente). El 18,1\% accede andando al territorio del Parque e incluye dentro de sus principales motivaciones de visita, la posibilidad de realizar algún tipo de actividad física (20,1\%), estar cerca de la naturaleza $(30,2 \%)$ o bien relajarse y desconectar $(14,0 \%)$. Acceden al parque principalmente por los accesos de Tavascan $(33,2 \%)$ y La Farga (16,1\%; figura 2$)$ y visitan otras zonas del parque durante su estancia en la zona (47,9\%) El 42,0\% concurre al parque durante el mes de Agosto. A diferencia del resto de grupos, son bastante críticos con la señalización del parque (casi el $27 \%$ la encuentra regular o incorrecta y el $18,1 \%$ sugiere mejorar este aspecto como principal observación de sugerencia o mejora). Para este segmento de visitantes, la necesidad de instaurar itinerarios interpretativos $(24,7 \%)$ es una prioridad por encima de los puntos de información (18,5\%) y los servicios de conservación (16,4\%). Al igual que el resto de grupos, no son partidarios de regular la recolección de setas $(51,1 \%)$, ni, en menor medida, la circulación motorizada al parque $(39,4 \%)$. Ver tablas 3,4 y 5 .

\section{Montañistas (ascensión a un pico)}

Este grupo, que conforma el 6,7\% del total de la muestra (107 visitantes), se caracteriza por ser el grupo que más conocimientos posee acerca del estatus de protección del Parque Natural $(92,5 \%)$ y, junto a los ciclistas, es uno de los grupos que más rasgos diferenciadores presenta respecto a la media del conjunto de visitantes. Así mismo, es el segundo grupo que más utiliza los itinerarios clásicos a la hora de visitar el parque $(90,7 \%)$, al tiempo que lo hace mayoritariamente por el acceso de la Farga $(62,7 \%)$ y, de una forma más recurrente respecto a la media, manifiesta conocer la zona a través de mapas, guías $u$ otras publicaciones especificas (12,1\%). Las principales motivaciones para visitar el parque son: estar cerca de la naturaleza $(27,7 \%)$, practicar algún tipo de actividad $f i$ sica $(21,4 \%) \mathrm{y}$, a diferencia del resto, visitar algún itinerario en particular (15,7\%). Además, suelen acudir al parque básicamente con amigos $(43,9 \%)$, en grupos de 3,8 personas, durante un tiempo superior al día de visita (el 37,4\% lo visita durante 2 - 3 días, con un valor medio de 25,1 horas), durante los meses de Agosto (35,5\%) y Septiembre $(26,2 \%)$ y de manera un tanto peculiar: más del $27 \%$ suele dormir en el territorio del Parque haciendo vivac o utilizando refugios de montaña $(14,1 \%)$, aunque también recurre a hoteles y campings $(23,6 \%)$. Cabe añadir además, que son el grupo que registra el nivel más alto de primera visita al área protegida $(41,1 \%)$ y el nivel más bajo de frecuentación de otras áreas del Parque Natural durante su estancia en la zona (el 72,9\% no suele visitar más de una zona del parque durante su estancia). Respec- 


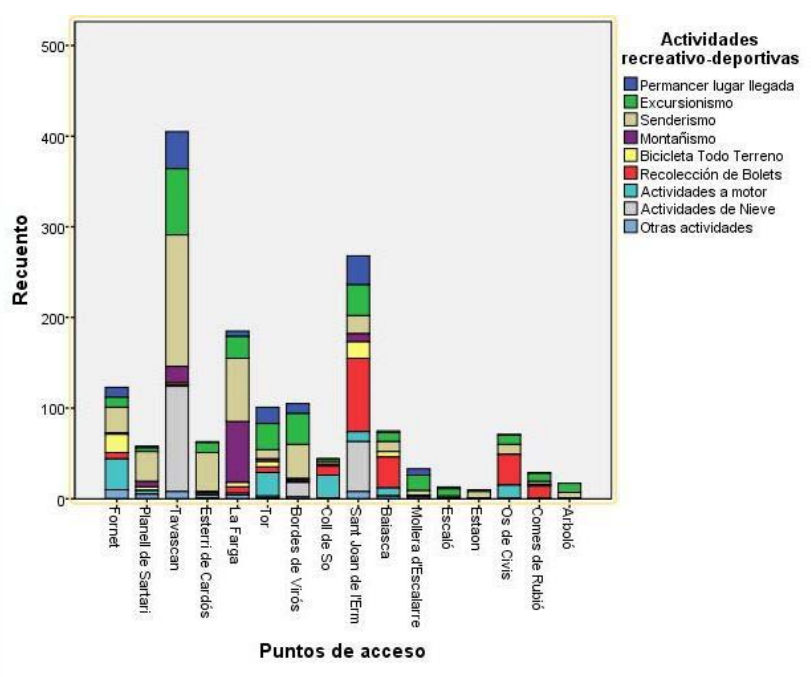

Figura 2. Actividades físico-deportivas versus puntos de accesos controlados

Figure 2. Physical activities versus access points

to a las opiniones y sugerencias de mejora, es uno de los grupos más críticos en cuanto a la oferta de las infraestructuras (aparcamientos, áreas recreativas, etc.), destacando el alto valor registrado en observaciones como: necesidad de mejorar la señalización (15\%) y de controlar la masificación (10,3\%). Finalmente, son los menos partidarios de regular la circulación motorizada (solo un $10,6 \%$ está a favor de instaurar algún tipo de control en esta actividad). Tablas 3, 4, 5 y figura 2.

\section{Ciclistas}

Con un total de 68 miembros (4,3\%), este grupo de visitantes, que practican la actividad de bicicleta todo terreno (BTT) o mountain bike (MTB) durante su visita al parque, se caracteriza por estar formado por gente mayoritariamente joven (38,5 años de media), con estudios universitarios $(56,1 \%)$, con el ratio más alto de hombres $(82,4 \%)$. Básicamente visitan el parque solos $(30,9 \%)$ o en grupo de amigos $(39,7 \%)$, y sobre todo, durante los meses de Julio (35,5\%) y Agosto (26,2\%). Ostentan la media más baja de integrantes por grupo (2,9 personas). Un $32,4 \%$ de los encuestados han visitado el área protegida entre 4 y 6 veces en los 2 últimos años (la frecuencia más alta de entre todos los visitantes) aunque es el grupo con menos conocimientos acerca del estatus de protección del parque (25\% no sabe nada al respecto). La principal motivación de visita es, a diferencia del resto de grupos, practicar algún tipo de actividad física o deportiva $(36,4 \%)$, seguida de estar cerca de la naturaleza $(22,8 \%)$, y relajarse y desconectar (14,2\%). Frecuentan itinerarios de tipo no clásicos $(16,2 \%)$ y suelen acceder al parque principalmente por entradas de uso minoritario como Fornet $(29,4 \%)$ y Sant Joan de l'Erm $(26,5 \%)$. Per- manecen en la zona entre medio día $(67,7 \%)$ y un día $(32,3 \%)$. Es uno de los grupos que más señalan la existencia de ciertos conflictos $(11,8 \%)$, al tiempo que menos críticos se muestran respecto a los folletos y mapas suministrados por el parque ( $25 \%$ los encuentra muy correctos) y al estado de limpieza y conservación de la zona (el $52,9 \%$ opina que está muy correcto). Son pro regulación del acceso motorizado al parque: más del $60 \%$ opina que hay que regularlo. Ver Tablas 3, 4, 5 y Figura 2.

\section{Recolector de setas}

Es el tercer grupo más grande en cuanto a número de personas, con un total de 199 integrantes (12,4\%). Tienen la media de edad más alta (52 años - el 56,8\% tiene más de 52 años), mayoritariamente son hombres $(73,4 \%)$ y se caracterizan por disponer del más bajo nivel de estudios (solo un 28,6\% tiene de tipo universitario) así como de conocimientos sobre el estatus de protección $(23,7 \%$ no lo conoce). Aunque la mayoría vienen de Barcelona $(51,8 \%)$, es el grupo con el porcentaje más alto de personas procedentes de los alrededores del parque o de la provincia de Lleida (35,7\% en total). Son también el segmento que más asiduamente visitan la zona (un $25,7 \%$ ha venido más de 7 veces en los 2 últimos años, y un 52,3\% más de 6 veces), el que más accede en coche $(95,5 \%)$ y el que, con gran diferencia, menos se aloja en la zona tras la visita (40,8\%). Suelen visitar el parque durante los meses de Agosto (19,1\%), Septiembre (37,7\%) y Octubre (15,6\%) y frecuentar, en un $91,5 \%$, los itinerarios de tipo clásico. Acceden al parque generalmente acompañados por amigos $(33,6 \%)$ o familiares $(30,2 \%)$, conocen la zona de toda la vida $(34,2 \%)$ y sus principales motivaciones son, evidentemente, recolectar setas $(43,8 \%)$ así como, estar cerca de la naturaleza (22,3\%) o relajarse y desconectar $(10,8 \%)$. Utilizan como acceso principal el de Sant Joan de l'Erm $(40,8 \%)$ y son uno de los grupos más críticos con el estado de limpieza (el 23,1\% lo encuentra regular o incorrecto) al tiempo que sugieren instaurar un servicio de conservación y control ambiental (25\%). Así mismo, son uno de los grupos menos partidarios de regular la recolección de setas (el 70,9\% opina que no hace falta, casi un $20 \%$ más que la media) y la circulación de vehículos motorizados $(5,8 \%)$. Curiosamente, es el que peor opinión tiene sobre el estado de masificación del parque (el 15,1\% opina que es excesiva). Tablas 3, 4, 5 figura 2.

\section{Motoristas (4x4, quads, motos)}

Los 131 integrantes que conforman este grupo de visitantes, que representan el $6,7 \%$ del total, se caracterizan por visitar el territorio del parque realizando travesías a través de caminos aptos para la circulación de vehículos $4 \times 4$, quads o motos. Residen de forma habi- 
tual mayoritariamente en las provincias de Barcelona $(50,9 \%)$, de Lleida $(11,5 \%)$ y en Andorra $(11,5 \%)$. Tienen una media de edad que ronda los 46 años y se caracterizan por acudir al parque, casi con idéntica proporción, con amigos o pareja $(32,8 \%$ y $32,1 \%$, respectivamente). El 35,1\% visita el parque durante el mes de Agosto. Así mismo, un gran volumen de ellos no se aloja en la zona $(24,3 \%)$, aunque otros recurren a las segundas residencias $(17,5 \%)$ u hoteles $(19,1 \%)$. Suelen realizar una visita fugaz, más del $85,6 \%$ visita el parque durante medio día, con una media inferior a 4 horas (valor más bajo registrado). Concurren al mismo en grupos reducidos de 3,5 personas, utilizando los puntos de acceso de Fornet (25,9\%), Tor (19,7\%) o Coll de So $(19,1 \%)$, estos dos últimos comunicados directamente a través de la frontera con Andorra. Visitan el parque para estar cerca de la naturaleza $(32,3 \%) \mathrm{y}$, en igual proporción, para visitar un itinerario en particular o estar con la familia o amigos (12,3\%). Al igual que el resto de visitantes, no son partidarios de regular la circulación de vehículos motorizados $(52,7 \%)$, ni la recolección de setas $(62,6 \%)$, en ambos casos un $10 \%$ más contundentes que el conjunto de visitantes. Los miradores panorámicos $(18,5 \%)$ y los puntos de información $(20,2 \%)$ son, para este colectivo, las principales demandas de infraestructuras y servicios a ofrecer por el parque natural. Ver Tablas 3, 4, 5 y Figura 2.

\section{Esquiadores y otros}

Este grupo de visitantes, que se dedican principalmente durante su visita al parque a practicar actividades de excursión con raquetas de nieve, esquí nórdico y, aunque en menor medida, esquí de montaña y esquí alpino, forman un grupo de 189 sujetos $(11,8 \%)$ y se caracterizan por ser jóvenes (casi el 60\% tienen entre 22 y 41 años), mujeres $(41,3 \%$, un $10 \%$ más que el valor medio) y poseer un alto nivel de estudios universitarios (52\%). Residen en Barcelona $(55,1 \%)$ y en una elevada proporción también en la zona (15,3\%). Acuden al parque durante los meses de Enero $(18,5 \%)$, Febrero (26,5\%), Marzo $(23,8 \%)$ y Diciembre $(30,2 \%)$, con los amigos o la familia $(69,4 \%)$. Presentan la segunda cifra más alta de visitantes por grupo $(5,1)$ y suelen alojarse en hoteles $(20,2 \%)$, en segundas residencias $(14,8 \%)$ o en albergues $(10,1 \%)$. Son el segundo grupo, después de los ciclistas, que visita el parque para hacer actividad física

\section{Discusión y conclusiones}

En términos generales la realización de este estudio ha permitido efectuar dos contribuciones importantes: una a nivel más global o general y otra a nivel más local o específico. A nivel global, la principal aportación de este estudio recae sobre la propuesta y justificación de una forma alternativa de organizar la información sobre los visitantes en los parques naturales, basada en una clasificación de las actividades recreativo-deportivas practicadas. Concretamente: permanecer en el lugar de llegada, excursionismo, senderismo, montañismo, ciclismo, recolección de setas, conducción de vehículos a motor y actividades de nieve. A nivel local, se ha facilitado información práctica y aplicable de gestión para el Parque Natural de l'Alt Pirineu, a fin de promover una mejor compatibilización de los usos y la conservación del espacio. La segmentación aquí propuesta es aplicable al 97\% de visitantes.

Se entiende que, los resultados del estudio y el enfoque sugerido cumplen con los supuestos propuestos por Kotler (1994) respecto a la identificación y la accesibilidad de los diferentes segmentos de la demanda. Esta agrupación, basada en la práctica de actividades recreativo-deportivas, reúne los requisitos para ser considerados como segmentos de mercado eficaces y apropiados; ya que la mayor parte de ellos son fáciles de identificar y de distinguir del resto de segmentos (vestimenta o requerimientos técnico-prácticos requeridos: calzado específico, mochila, canasta, etc. concentración de usos en determinados puntos, etc.) y se basan en una característica que mantiene una cierta homogeneidad interna (presencia de diferencias significativas en la mayor parte de los aspectos estudiados).

Los segmentos identificados en este estudio fueron ocho: paseantes, excursionistas, senderistas, montañistas, ciclistas, recolectores de setas, motoristas y esquiadores. A pesar de que estos segmentos pueden ser diferentes en otros contextos geográficos de acuerdo a las características geográficas y los planes de uso, la segmentación de visitantes basada en la práctica de actividades recreativo-deportivas puede beneficiar también a otros espacios naturales protegidos. Sirvan de ejemplo los resultados obtenidos por Múgica (1993) en el estudio de las preferencias paisajísticas de los visitantes de los Parques Nacionales del Teide, Doñana, Ordesa y Monte Perdido y Covadonga, que a pesar de no coincidir plenamente con la propuesta de categorización de actividades recreativo-deportivas aquí presentada (la agrupación propuesta en dicho estudio fue: permanecer en el lugar de llegada $-14,3 \%$-, paseos cortos $-22,4 \%$-, paseos de menos de 30 minutos $-16,6 \%$, paseos de menos de menos de 3 horas $-25,3 \%$-, recorridos largos $-6,6 \%$ - y visita guida $-7,8 \%$-), sí que permite una extrapolación de casi el $93 \%$ de los visitantes estudiados, con excepción de los que realizaban visitas guiadas.

En general, los resultados demuestran la presencia de ciertos patrones de comportamiento de acuerdo con la actividad practicada, aunque en algunos grupos más que otros. Al respecto, la tendencia parece resultar bastante clara: a mayor especificidad o requerimiento físico-técnico de la actividad practicada, mayor diferenciación.

En este sentido, vale la pena apuntar que, la segmentación basada en las actividades recreativo-deportivas, tiene ventajas adicionales que en algunos casos pueden ir 
más allá de la gestión específica del espacio natural protegido en cuestión, tales como:

1. La información disponible también puede ser útil a la hora de negociar con los representantes, asociaciones o federaciones sobre la regulación de determinados tipos de prácticas; extensible, en algunos casos, a otras zonas similares o colindantes. Ej. Recolección de setas, escalada en roca, etc.

2. El conocimiento de la demanda actual de este tipo de actividades, además de facilitar el seguimiento de las mismas, puede ayudar a promocionar aquellas actividades más compatibles con las necesidades de conservación del espacio, o a canalizar las actividades con más impacto hacia zonas menos frágiles del área protegida.

3. En la mayor parte de los casos, la demanda de este tipo de actividades puede estar asociada a impactos ambientales específicos tales como los relacionados por: NPCA (1992a y b), Wilson \& Seney (1994), Leung \& Marion (1996 y 2000), Thurston \& Reader (2001), Lynn \& Brown (2003), Wenjun et al. (2005), White et al. (2006), Davies \& Newsome (2009), Pickering et al. (2010). Por lo tanto, el conocimiento de los diferentes segmentos puede ayudar a optimizar el diseño y puesta en marcha de programas específicos de minimización de impactos, tanto en el parque como en zonas externas al territorio protegido.

Concretamente se puede hablar de la puesta en marcha de programas de sensibilización e información que den a conocer: la importancia del uso del trazado principal de la red de senderos en el caso de los excursionistas, senderistas o montañistas; la conveniencia de no utilizar determinadas zonas en función de la climatología o estación del año en el caso de los ciclistas (zonas con pendiente pronunciada en épocas de lluvia), o las importantes consecuencias ambientales que una mala praxis en la recolección de setas puede ocasionar sobre el medio en el que se desarrollan.

A nivel local, este estudio ha permitido constatar como, por ejemplo, los paseantes (identificables en gran medida por el uso de un tipo de vestimenta poco técnica, sobre todo en lo que al calzado se refiere) son los que visitan el parque en grupos más numerosos (5,2 persona/ grupo) y acceden al parque por los puntos de entrada más frecuentados (Tavascan y San Joan de l'Erm), aunque no visitan el territorio del Parque Natural propiamente dicho $(47,4 \%)$. O que el grupo de los senderistas y montañistas (que suelen ir equipados con botas de montaña, mochilas y, en gran medida, con bastones tipo esquí nórdico) son los que más conocimiento poseen sobre el estatus de protección de la zona (más del $85 \%$ en este caso), al tiempo que son unos de los segmentos de visitantes que más impacto económico pueden llegar a generar en la zona. Más del 55\% utiliza un alojamiento de pago, permanecen en la zona una media superior a los tres días; que en el caso de los senderistas asciende a los 5,5 días, y frecuentan con más asiduidad otras zonas del territorio $(72,9 \%)$.

Destacan también, en este sentido, el grupo de ciclistas (identificables fácilmente gracias al uso de la bicicleta), por su limitado nivel de conocimiento sobre el estatus de protección de la zona $(25 \%)$, por su elevada frecuentación de itinerarios no clásicos $(16,4 \%)$ y su amplio apoyo a la necesidad de regular el acceso motorizado y la recolección de setas. Como así también por ser el grupo con el valor medio de personas por grupo más reducido (2,9 personas/grupo). O el grupo de recolectores de setas (identificables por el uso de canastas $u$ otro tipo de sistemas de recolección), caracterizados por ser el grupo con más edad (media de edad superior a 52 años), menor nivel educativo y de conocimiento sobre el estatus de protección de la zona (casi un $24 \%$ no lo conoce), que durante menos tiempo visita la zona (2,2 días de media) y menos uso hace de los alojamientos de pago (menos del 24\% utiliza alojamientos de pago). Así mismo, es uno de los segmentos que más frecuenta el parque (52,3\% lo vista más de 4 veces al año) y más crítico se manifiesta respecto a la masificación y estado de limpieza y conservación del parque.

Sin olvidar al resto de grupos, que aun teniendo un perfil menos diferenciado, también muestran ciertas particularidades que merecen la pena reconocer para una mejor gestión del uso público de este espacio.

A modo de ejemplo, estas son algunas de las recomendaciones que se pueden realizar:

1. La puesta en marcha de campañas de información respecto al estatus de protección centrada en los segmentos de recolectores de setas y ciclistas, puesto que estos son los dos grupos que muestran un nivel más bajo de conocimiento sobre el estatus de protección del parque al tiempo que son unos de los que visitan la zona con más frecuencia (recolectores de setas), de manera más estacional (ambos grupos) y mediante el uso de rutas no clásicas (ciclistas). En este sentido, el punto de acceso de San Joan de l'Erm podría ser un buen lugar para el lanzamiento de esta campaña, ya que es la entrada principal de ambos segmentos (Figura 2).

2. La promoción del uso del parque entre los senderistas y montañistas, que además de ser unos de los grupos con más nivel educativo y mejor conocimiento sobre el estatus de protección, presentan un comportamiento de visita apto o recomendable para este tipo de espacios (tipo de itinerario que frecuenta, duración de visita, número de integrantes por grupo, etc.).

Al respecto vale la pena destacar que estos dos grupos son unos de los segmentos que más impacto económico generan en la zona, ya sea por el tipo de alojamiento que utiliza, ya sea por el tiempo de visita o por la frecuentación de otras zonas del parque durante su visita. Una buena forma de preparar el terreno para esta promoción sería trabajar en la mejora de algunas de las principales reivindicaciones 
de este grupo, como son: la señalización de los diferentes itinerarios, la adecuación y mantenimiento de las principales pistas de acceso, así como un mayor control sobre la masificación en la zona, sobre todo durante los meses de verano, coincidiendo con la punta de afluencia de estos dos grupos de visitantes.

3. El diseño e implementación en las zonas de acceso de itinerarios interpretativos que invitasen al segmento de paseantes a realizar pequeñas excursiones que los adentren en el territorio del parque y acerquen al conocimiento de algunas de las características principales de este. El hecho de que el grupo de paseantes comparta idénticas motivaciones de visita con los excursionistas, aunque priorizadas de forma diferente, es un buen argumento para pensar que esta canalización puede ser posible. Para ello, los meses de Enero y Abril podrían ser un buen momento para la puesta en marcha y evaluación de la eficacia de las medidas adoptadas, por el hecho de acoger un buen número de visitantes de este segmento sin coincidir con la frecuentación punta de este espacio.

En relación a estas dos últimas recomendaciones, relativas a la promoción del uso del parque por parte de los segmentos de paseantes (implementación de itinerarios interpretativos), o de excursionistas y montañistas (incremento del uso), es imprescindible valorar muy detenidamente la inducción de nuevos o mayores impactos ambientales. En este sentido, la denudación del suelo y el incremento de residuos son dos de los impactos claves a vigilar. Al respecto, sería interesante considerar la puesta en marcha de estas acciones una vez iniciadas las correspondientes campañas de información, educación, sensibilización, etc.

Finalmente, se ha de señalar como posible línea futura de trabajo la aplicación de esta propuesta de segmentación, basada en las actividades recreativo-deportivas, en otros parques o áreas naturales. Nuevos estudios podrían determinar si dicha segmentación puede también ser considerada como una segmentación óptima de gestión en otros espacios naturales protegidos.

A nivel local, la investigación futura podría estar encaminada a conocer la efectividad de las campañas de información, educación, sensibilización, promoción, canalización, minimización de impactos, etc. de los diferentes segmentos de visitantes, o el seguimiento de la afluencia de estos en función de las circunstancias climáticas de las diferentes estaciones del año. Así mismo, otra línea de investigación podría centrarse en extrapolar los datos presentados en este trabajo a una agrupación según los planes sectoriales de promoción del turismo por valles, actualmente ya en marcha. Estamos convencidos de que estos son temas muy interesantes y potencialmente muy beneficiosos en la mejora de la gestión de este parque, pero también de otros espacios naturales protegidos españoles. Esperamos despertar desde estas líneas la curiosidad de otros investigadores interesados en esta temática.

\section{Agradecimientos}

A todo el personal del Parque Natural, con una mención especial a Jordi Palau (director) y a los técnicos Marc Garriga, Moisès Villanueva y Eva Sanchís. Este proyecto fue financiado por la Generalitat de Catalunya. Departament d'Agricultura, Ramaderia, Pesca, Alimentació i Medi Rural. Parc Natural de l'Alt Pirineo.

\section{Referencias}

Autònoma de Barcelona Federació d'Entitats Excursionistes de Catalunya (FEEC). Disponible en: Universitat de Barcelona. http://www.ub.edu/sbcn2011/docs/sbcn2011_resumen informe_PNMM.pdf

Arnberger, A., Eder, R., Allex, B., Sterl, P. \& Burns, R. C., 2012. Relationships between national-park affinity and attitudes towards protected area management of visitors to the Gesaeuse National Park, Austria. Forest Policy and Economics, 19: 48-55. http://dx.doi.org/10.1016/j.forpol.2011.06. 013.

Atauri, J., Bravo, M. \& Ruiz, A., 2000. Visitors' landscape preferences as a tool for management of recreational use in natural areas: A case study in Sierra de Guadarrama (Madrid, Spain). Landscape Research, 25(1): 49-62. http://dx.doi. org/10.1080/014263900113163

Benayas, J., 2000. Manual de buenas prácticas del monitor de naturaleza: espacios naturales protegidos de Andalucía. Consejería de Medio Ambiente. Junta de Andalucía. ISBN 84-89650-87-X

Bricker, K.S. \& Kerstetter, D.L., 2000. Level of specialization and place attachment: an exploratory study of white water recreationists. Leisure Sciences: an Interdisciplinary Journal, 22 (4): 233-257. http://dx.doi.org/10.1080/01490409950202285

Brown, P. \& Haas, G., 1980. Wilderness recreation experiences. The Rawah case. Journal of Leisure Research, 12: 229-241. Disponible en: CAB direct http://www.sagamorepub.com/ products/journal-leisure-research

Cessford, G.R., 2003. Perception and reality of conflict: Walkers and mountain bikes on the Queen Charlotte Track in New Zealand. Journal for Nature Conservation, 11(4): 310-316. http://dx.doi.org/10.1078/1617-1381-00062

Collins, R. \& Hodge, I., 1984. Clustering visitors for recreation management. Journal of Environmental Management, 19(2): 147-158.

Chhetri, P., Arrowsmith, C. \& Jackson, M., 2004. Determining hiking experiences in nature-based tourist destinations. Tourism Management, 25: 31-43. http://dx.doi.org/10.1016/ S0261-5177(03)00057-8

Chi-Ok, O.H. \& Ditton, R.B., 2006. Using recreation specialization to understand multi-attribute management preferences. Leisure Sciences: an Interdisciplinary Journal, 28 (4): 369 384. http://dx.doi.org/10.1080/01490400600745886

Davies, C. \& Newsome, D., 2009. Mountain bike activity in natural areas: impacts, assessment and implications for management: a case study from John Forrest National Park, Western Australia. Australia. Sustainable Tourism CRC. ISBN: 9781921658044 (pbk.) 9781921658549 (pdf).

De Lucio, J.V. \& Múgica, M., 1994. Landscape preferences and behaviour of visitors to Spanish national parks. Landscape and Urban Planning, 29: 145-160. http://dx.doi.org/ 10.1016/0169-2046(94)90024-8

Dudley, N., 2008. Guidelines for applying protected area management categories. Gland, Switzerland. Retrieved from http://data.iucn.org/dbtw-wpd/edocs/paps-016.pdf 
EUROPARC-ESPAÑA, 2008. Anuario Europarc-España del estado de los espacios naturales protegidos 2007 [EuroparcSpain Yearbook: The status of Protected Natural Areas 2007]. Madrid: Fundación Fernando González Bernáldez, Madrid, 143 pp. Disponible en: http://www.redeuroparc.org/documentos_anexos/Publicaciones/Anuario/anuario2007.pdf

Farías, E.I., 2000. El aprovechamiento recreativo, deportivo y turístico de los espacios naturales protegidos. Modelos de Frecuentación. El caso del Parc Nacional d'Aigüestortes $i$ Estany de Sant Maurici. [Recreation, sport and tourism leveraging of protected natural areas. Frequentation models. The case of the Parc National d'Aigüestortes i Estany de Sant Maurici]. Institut Nacional d'Educació Física de Catalunya. Universitat de Lleida, Lleida. Disponible en: TDX http://hdl.handle.net/10803/8061

Farías, E.I., Grau, H.R. \& Camps, A., 2005. Trail preferences and visitor characteristics in Aigüestortes i Estany Sant Maurici National Park, Spain. Mountain Research and Development, 25(1): 51-59. http://dx.doi.org/10.1659/02764741(2005)025[0051:TPAVCI]2.0.CO;2

Farías, E.I., Tisone, J., Hernandez, M.E., Vila, G., Segarra, G. \& Sala, E., 2010. Uso recreativo, deportivo y turístico de la Sierra de San Javier. Bases para su planificación. En: Grau, H. R (Ed.) Ecología regional de una interface natural-urbana. EDUNET, pp 191-214. ISBN 978-987-1366-70-S

Farías, E.I., 2011a. Managing for recreational experiencies opportunities: the case of hikers in protected areas in Catalonia, Spain. Environmental Management, 47 (3): 482-496. http://dx.doi.org/10.1007/s00267-010-9606-z

Farías, E.I., 2011b. Estudi sobre l'afluència i caracterització dels visitantes al Parc Natural de l'Alt Pirineu [Visitors flows and characterization of people at the Natural Park of the High Pyrenees]. Generalitat de Catalunya. Departament de Medi Ambient. Disponible: http://www20.gencat.cat/ portal/site/parcsnaturals/menuitem.cca3e077efe101130161f ea3b0c0e1a0/?vgnextoid=9982280f35f42210VgnVCM100 $0008 \mathrm{~d} 0 \mathrm{c} 1 \mathrm{e} 0 \mathrm{aRCRD} \&$ vgnextchannel $=9982280 \mathrm{f} 35 \mathrm{f} 42210 \mathrm{~V}$ gnVCM1000008d0c1e0aRCRD\&vgnextfmt=detall\&conten tid=9b1ab12c6d3e6310VgnVCM1000008d0c1e0aRCRD

Frochot, I., 2005. A benefit segmentation on tourists in rural areas: a Scottish perspective. Tourism Management, 26: 335-346. http://dx.doi.org/10.1016/j.tourman.2003.11.013

Gómez-Limón, F.J., 1996. Uso recreativo de los Espacios Naturales. Frecuentación, factores explicativos e impactos asociados. El caso de la comunidad de Madrid. Tesis Doctoral. Facultad de Ciencias. Departamento Interuniversitario de Ecología. Universidad Autónoma de Madrid. ISBN: 84-89198-23-3

Gómez-Limón, F. J., Medina, L., Atance, I. \& Garrido, A., 2002. Los visitantes de la comarca de Doñana. Fundación Fernando González Bernáldez. Europarc-España. Disponible en: http://www.conozcadonana.com/arch_var/monografico4. pdf

Gruber, G. \& Benayas, J., 2002. Diagnóstico de los planes de uso público en los espacios naturales protegidos españoles. La investigación y el seguimiento en los espacios naturales protegidos del siglo XXI. Diputación de Barcelona. Barcelona. ISBN: 84-7794-884-4

Hockings, M., Stolton, S. \& Dudley, N., 2002. Evaluating Efectivess. A summary for park managers and policy makers. Universitat de Queensland. UICN. Australia. Disponible en: http://planet.botany.uwc.ac.za/NISL/Conservation\%20Biology/Fifth World Parks congress Durban 2003/ stream\%20outputs/Background\%20Documents/EME $\% 20$ Summary\%20Paper.pdf

Kotler, P., 1994. Marketing management. Analysis, planning, implementation, and control. New Yersey: Prentice-Hall. ISBN: 10:0137228511
Kuentzel, W.F. \& Heberlein, T.A., 1992. Does specialization affect behavioural choices and quality judgments among hunters? Leisure Sciences: an Interdisciplinary Journal, 14(3): 211-226. http://dx.doi.org/10.1080/01490409209513169

Leung, Y.F. \& Marion, J.L., 1996. Trail degradation as influenced by environmental factors: a state-of-the-knowledge review. Journal of Soil and Water Conservation 51 (2): 130-136.

Leung, Y.F. \& Marion, J.L., 2000. Recreation impacts and management in wilderness: A state of knowledge review. En: Cole, D.N., McCool, S.F., Borrie, W.T. \& O'Loughlin, J., (Eds.) Wilderness Science in a Time of Change: Vol. 5. Wilderness Ecosystems, Threats, and Management (pp. 23-48). Ogden, US: USDA Forest Service. Disponible en: http:// www.fs.fed.us/rm/pubs/rmrs_p015_1.pdf

Lynn, N.A. \& Brown, R.D., 2003. Effects of recreational use impacts on hiking experiences in natural areas. Landscape and Urban Planning, 64: 77-87. http://dx.doi.org/10.1016/ S0169-2046(02)00202-5

Marion, J.L., 2006. Assessing and understanding trail degradation: results from Big South Fork National River and Recreational Area. USDA: National Park Service.

McÇoll, S.F. \& Reilly, M., 1993. Benefit segmentation analysis of state Park visitor setting preferences and behavior. Journal of Park and Recreation Administration, 1-14. Disponible en: CAB direct http://www.cabdirect.org/abstracts/ 19941806012.html

Merrill, K. \& Graefe, A., 1998. The relationship between activity specialization and preferences for setting and route attributes of selected rock climbers. En Vogelsong, H. G. (Ed) Proceedings of the 1997 Northeastern Recreational Research Symposium (pp. 40-43). Radnor, US: Department of Agriculture, Forest Service. Disponible en: http://www.esf. edu/nerr/past/1997.pdf

Mowen, A.J., Trauntvein, N.E., Graefe, A.R. \& Son, J.S., 2012. The influence of visitor characteristics on state park physical activity levels. Journal of park and Recreation Administration, 30 (2).

Múgica, M., 1993. Modelos de demanda paisajística y uso recreativo de los espacios naturales [Lanscape demand models and recreational use of natural areas]. PhD thesis. Facultad de Ciencias. Departamento Interuniversitario de Ecología. Universidad Autónoma de Madrid, Madrid. ISBN 84-89198-15-S

Muñoz, M., 2008. Evaluación y financiación del uso público en espacios naturales protegidos. El caso de la Red Española de Parques Nacionales. [Assessment and funding of public use in protected areas. The case of the Spanish Network of National Parks] PhD thesis. Departamento Interuniversitario de Ecología. Universidad Autónoma de Madrid, Madrid.

Newsome, D., More, S.A. \& Dowling, R.D., 2002. Aspects of tourism. Natural area tourism. Ecology, impacts and management. Channel view publications. ISBN: 1-853150-25-3 (hbk)

National Parks Conservation Association (NPCA), 1992a. Visitor Impact Management. A review of research. Volume one. USDA: National Park Service.

National Parks Conservation Association (NPCA), 1992b. Visitor Impact Management. The planning. Volume two. USDA: National Park Service.

Organismo Autónomo de Parques Nacionales (OAPN), 2011. La red de parques naciones en la sociedad. Estudio Explicativo sobre la percepción social de la Red de Parques Nacionales. Cuadernos de la Red de Parques Nacionales. 71 pp. ISBN: 978-84-8014-811-5

Pickering, C.M., Hill, W., Newsome, D., Leung, Y. F., 2010. Comparing hiking, mountain biking and horse riding impacts on vegetation and soils in Australia and the United States of 
America. Journal of Environmental Management, 91: 551562. http://dx.doi.org/10.1016/j.jenvman.2009.09.025

Pouta, E., Sievänen, T., Neuvonen, M., 2004. Profiling recreational users of national parks, national hiking areas and wilderness areas in Finland. En: Sievänen, T., Erkkonen, J., Jokimäki, J., Saarinen, J., Tuulentie, S. \& Virtanen, E. (Eds.). Policies, Methods and Tools for Visitor Management. Proceedings of the Second International Conference on Monitoring and Management of Visitor Flows in Recreational and Protected Areas. (pp. 357-364). Rovaniemi, Finland: Työraportteja. Disponible en: http://www.metla.fi/julkaisut/ workingpapers/2004/mwp002.htm

Priskin, J., 2003. Tourist perceptions of degradation caused by coastal nature-based recreation. Environmental Management, 32(2): 189-204.http://dx.doi.org/10.1080/01490409509513254

Rollins, R.B. \& Rouse, J., 1992. Segmenting backcountry visitors by setting preferences. En: Willison, J.H.M., BondrupNielsen, S.; Drysdale, C., Herman, T.B., Munro, N.W.P., \& Pollock, T.L. Science and the management of protected areas (pp. 485-497). New York: Acadia University, Elsevier. ISBN: 0444-89163-3

Schmitz, M.F., Aranzabal, I. \& Pineda, F.D., 2007. Spatial analysis of visitor preferences in the outdoor recreational niche of Mediterranean cultural landscape. Environmental Conservation, 34: 300-312. http://dx.doi.org/10.1017/ S0376892907004249

Schuett, M.A. \& Pierskalla, C.D., 2007. Managing for desired experiences and site preferences: The case of fee-fishing anglers. Environmental Management, 39(2): 164-177. http:// dx.doi.org/10.1007/s00267-005-0366-0

Schreyer, R., 1982. Experience level affects expectations for recreation participation. En: Lime, D.W. (Ed.) Forest and river recreation: Research Update. St. Paul, MN: University of Minnesota.

Siegel, S. \& Castellan, N. J., 1988. Non-parametric statistics for the behavioural sciences. $2^{\text {nd }}$ ed. Mc Grau-Hill, New York. 399 pp. ISBN: 13 978-0070573574
Thurston, E. \& Reader, R.J., 2001. Impacts of experimentally applied mountain biking and hiking on vegetation and soil of a deciduous forest. Environmental Management, 27: 397409. http://dx.doi.org/10.1007/s002670010157

Virden, R.J. \& Schreyer, R., 1988. Recreation specialization as an indicator of environmental preference. Environmental and Behaviour, 20(6): 721-739. http://dx.doi.org/10.1177/ 0013916588206004

Wall Reinius, S. \& Fredman, P., 2007. Protected areas as attractions. Annual of Tourism Research, 34 (4): 839-854. http:// dx.doi.org/10.1016/j.annals.2007.03.011

Wallace, G.N. \& Smith, M.D., 1997. A comparison of motivations, preferred management actions, and setting preferences. Journal of Park and Recreation Administration, 15: 5982. Disponible en: Journals http://js.sagamorepub.com/jpra/ article/view/1698

Wenjun, L., Xiadong, G.E. \& Chunyan, L., 2005. Hiking trails and tourism impact assessment in protected area: Jiuzhaigou biosphere Reserve, China. Environmental Monitoring and Assessment, 108:279-293.http://dx.doi.org/10.1007/s10661005-4327-0.

White, D.D., Waskey, M.T., Brodehl, G.P. \& Foti, P.E., 2006. A comparative study of impacts to mountain bike trails in five common ecological regions of the south-western U.S. Journal of Park and Recreation Administration, 24(2): 21-41. Disponible en: http://imba.org.uk/wp-content/uploads/white_et_al_study.pdf

Wilson, J.P. \& Seney, J.P., 1994. Erosional impacts of hikers, horses, motors cycles, and off-road bicycles on mountain trails in Montana. Mountain Research and Development, 14: 77-88. Disponible en: http://132.198.101.197/ snrvtdc/ trails/erosionalimpactofhikers.pdf

Woratschek, H., Hannich, F.M. \& Ritchie, B., 2007. Motivations of physicals tourists - an empirical analysis in several European rock climbing regions. Disponible en: Universität Bayreuth Aktuell http://www.fiwi.uni-bayreuth.de/de/download/WP_02-07.pdf. 